\title{
Renormalization of circle diffeomorphisms with a break-type singularity $]^{3}$
}

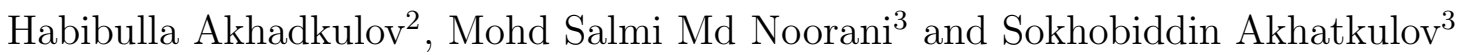

\begin{abstract}
Let $f$ be an orientation-preserving circle diffeomorphism with irrational rotation number and with a break point $\xi_{0}$, that is, its derivative $f^{\prime}$ has a jump discontinuity at this point. Suppose that $f^{\prime}$ satisfies a certain Zygmund condition dependent on a parameter $\gamma>0$. We prove that the renormalizations of $f$ are approximated by Möbius transformations in $C^{1}$-norm if $\gamma \in(0,1]$ and they are approximated in $C^{2}$ norm if $\gamma \in(1,+\infty)$. It is also shown, that the coefficients of Möbius transformations get asymptotically linearly dependent.
\end{abstract}

\section{Introduction and results}

One of the most studied classes of dynamical systems are orientation-preserving diffeomorphisms of the circle $\mathbb{S}^{1}=\mathbb{R} / \mathbb{Z}$. Poincaré (1885) noticed that the orbit structure of orientation-preserving diffeomorphism $f$ is determined by some irrational mod 1 , called the rotation number of $f$ and denoted $\rho=\rho(f)$, in the following sense: for any $\xi \in \mathbb{S}^{1}$, the mapping $f^{j}(\xi) \rightarrow j \rho \bmod 1, j \in \mathbb{Z}$, is orientation-preserving. Denjoy proved, that if $f$ is an orientation-preserving $C^{1}$-diffeomorphism of the circle with irrational rotation number $\rho$ and $\log f^{\prime}$ has bounded variation then, the orbit $\left\{f^{j}(\xi)\right\}_{j \in \mathbb{Z}}$ is dense and the mapping $f^{j}(\xi) \rightarrow j \rho \bmod 1$ can therefore be extended by continuity to a homeomorphism $h$ of $\mathbb{S}^{1}$, which conjugates $f$ to the rigid rotation $f_{\rho}: \xi \rightarrow \xi+\rho \bmod 1$.

In this context, a natural question is to ask under what condition one can obtain the smoothness of conjugacy $h$. The first local results, that is the results requiring the closeness of diffeomorphism to the rigid rotation, were obtained by Arnold [2] and Moser 25]. Next Herman [5] obtained a first global result (i.e. not requiring the closeness of diffeomorphism to the rigid rotation) asserting regularity of conjugacy of the circle diffeomorphism. Further, his result was developed by Yoccoz [31], Stark [26], Khanin \& Sinai [13, 14, Katznelson \& Ornstein [8, 9] and Khanin \& Teplinsky [17]. They have shown that if $f$ is $C^{3}$ or $C^{2+\nu}$ and $\rho$ satisfies a certain Diophantine condition then the conjugacy will be at least $C^{1}$.

Note, the renormalization approach in [14] and [26] to Herman's theory is more natural. In this approach, the regularity of conjugacy statement can be obtained using the convergence of renormalizations of sufficiently smooth circle diffeomorphisms with the same irrational rotation number. In fact, the renormalizations of circle diffeomorphisms approach to a family of linear maps with slope 1 . Such convergence implies the regularity of conjugacy if the rotation number satisfies the certain Diophantine condition.

A natural generalization of diffeomorphisms of the circle are diffeomorphisms with break points, i.e., those circle diffeomorphisms which are smooth everywhere with the

\footnotetext{
${ }^{1}$ MSC2000: 37C15, 37C40, 37E10, 37F25. Keywords and phrases: circle diffeomorphism, break point, rotation number, renormalization, fractional-linear maps, convergence.

${ }^{2}$ School of Quantitative Sciences, University Utara Malaysia, CAS 06010, UUM Sintok, Kedah Darul Aman, Malaysia. E-mail: akhadkulov@yahoo.com

${ }^{3}$ School of Mathematical Sciences Faculty of Science and Technology University Kebangsaan Malaysia, 43600 UKM Bangi, Selangor Darul Ehsan, Malaysia. E-mail: msn@ukm.edu.my (M.S. Noorani), akhatkulov@yahoo.com (S. Akhatkulov).
} 
exception of finitely many points at which their derivatives have jump discontinuities. Circle diffeomorphisms with breaks were investigated by Herman [5] in the piecewiselinear (PL) case. General (non PL) circle diffeomorphisms with a break point was studied by Khanin \& Vul [18, 28] at the beginning of 90's.

It turns out that, the renormalizations of circle diffeomorphisms with break points are rather different from those of smooth diffeomorphisms. Indeed, the renormalizations of such diffeomorphisms approach exponentially to a two-dimensional space of Möbius transformations (see Theorem 1.1) with very non-trivial dynamics. In the sequel we provide some basic utilizations of Khanin \& Vul's [28] result in the rigidity theory. Rigidity, in this context, is the phenomenon of smooth conjugacy between any two maps which a priori are only topologically equivalent.

Since the renormalizations of diffeomorphisms with breaks, approach to a two-dimensional space of Möbius transformations, it is convenient to study the action of renormalization operator on a space of pairs (for particular size of a break) in $\mathbb{R}^{2}$ which correspond to Möbius pairs. The investigations of those Möbius transformations in [10], [16] and [19] showed that the renormalization operator possesses strong hyperbolic properties in a certain domain of that space, which are analogous to those predicted by Lanford [21] in case of critical rotations.

The hyperbolicity of renormalization operator and as well as analysing the Möbius transformations give exponential convergence of renormalizations of two circle diffeomorphisms with a break in $C^{2}$-topology [10], [16. Very recently, the renormalization conjecture, that is any two smooth circle diffeomorphisms with a break, with the same irrational rotation number and same size of the break, belong to the same universality class, (i.e., their renormalizations approach each other) has been proven by Khanin \& Kocić [11. This conjecture for $C^{\infty}$-critical circle maps with bounded type of rotation numbers was proven by de Faria \& de Melo [22, 23] and extended in the analytic setting, to all rotation numbers by Yampolsky [30. Another aspect of Khanin \& Vul's result is, their result plays an important role to show uniformly regularity (see definition [12, [16]) of renormalizations.

Hence, the validity of above statements ensure $C^{1}$-rigidity of circle maps with singularities. Note that, the rigidity results for circle diffeomorphisms with breaks have been obtained by Khanin \& Khmelov [10] for a countable set of irrational rotations numbers and for a zero measure set of irrational rotations numbers by Khanin \& Teplinsky [16]. The rigidity problem was recently completely solved by Khanin et al. [12] for almost all rotation numbers. In the case of critical circle maps de Faria \& de Melo [22, 23] proved that for a full measure set of irrational rotation numbers, $C^{3}$-smooth critical maps with odd integer order of the critical point, the conjugacy is $C^{1+\alpha}$ for some $\alpha>0$. And any two real-analytic critical circle maps with the same irrational rotation number of bounded type are $C^{1+\nu}$ conjugate for some $\nu>0$. Recently, Khanin \& Teplinsky [15] proved that for real-analytic critical circle maps and for any irrational rotation numbers the rigidity holds, this rigidity is called robust rigidity.

The purpose of this work is to extend Khanin \& Vul's result [28]. For this we consider a class of circle diffeomorphisms with break points satisfying a certain Zygmund condition depending on a parameter $\gamma>0$. The class of such diffeomorphisms is wider than $C^{2+\nu}$. In our first main Theorem 1.2 we show that, if $\gamma \in(0,1]$ then the renormalizations approach to Möbius transformations with the rate of $\mathcal{O}\left(n^{-\gamma}\right)$ in $C^{1}$-topology. In the case of $\gamma \in(1,+\infty)$ this class is a subset of $C^{2}$, therefore we investigate the renormalizations in $C^{2}$-topology. In the second main Theorem 1.3 we show that, if $\gamma \in(1,+\infty)$ then the renormalizations approach to Möbius transformations with the rate of $\mathcal{O}\left(n^{-\gamma}\right)$ in $C^{1}$ - 
topology. Moreover, the second derivative of the renormalizations approach to the second derivative of those Möbius transformations with the rate of $\mathcal{O}\left(n^{-(\gamma-1)}\right)$ in $C^{0}$-topology. It is also shown that, the coefficients of Möbius transformations get asymptotically linearly dependent.

\subsection{Renormalizations of circle diffeomorphisms with a break point}

Let $f: \mathbb{S}^{1} \rightarrow \mathbb{S}^{1}$ be a circle diffeomorphism with a single break point $\xi_{0}$ i.e., $f$ satisfies the following conditions:

(i) $f \in C^{1}\left(\left[\xi_{0}, \xi_{0}+1\right]\right)$;

(ii) $\inf _{\xi \neq \xi_{0}} f^{\prime}(\xi)>0$;

(iii) $f$ has one-sided derivatives $f^{\prime}\left(\xi_{0} \pm 0\right)>0$ and

$$
c:=\sqrt{\frac{f^{\prime}\left(\xi_{0}-0\right)}{f^{\prime}\left(\xi_{0}+0\right)}} \neq 1 .
$$

The number $c$ is called a size of break of $f^{\prime}$ at $\xi_{0}$. Below we briefly recall the definition of renormalization and formulate some obtained results. Let the rotation number $\rho$ be an irrational. We use the following continued fraction expansion of rotation number

$$
\rho=1 /\left(k_{1}+1 /\left(k_{2}+\ldots\right)\right):=\left[k_{1}, k_{2}, \ldots, k_{n}, \ldots\right) .
$$

The sequence of positive integers $\left(k_{n}\right)$ with $n \geq 1$ called partial quotients and it is infinite if and only if $\rho$ is irrational. Every irrational $\rho$ defines uniquely the sequence of partial quotients. Conversely, every infinite sequence of partial quotients defines uniquely an irrational number $\rho$ as the limit of the sequence of rational convergents $p_{n} / q_{n}=\left[k_{1}, k_{2}, \ldots, k_{n}\right]$. The coprime numbers $p_{n}$ and $q_{n}$ satisfy the recurrence relations $p_{n}=k_{n} p_{n-1}+p_{n-2}$ and $q_{n}=k_{n} q_{n-1}+q_{n-2}$ for $n \geq 1$, where, for convenience we set $p_{0}=0, q_{0}=1$ and $p_{-1}=1, q_{-1}=0$. Taking the break point $\xi_{0} \in \mathbb{S}^{1}$, we define the $n$th fundamental segment $I_{0}^{n}:=I_{0}^{n}\left(\xi_{0}\right)$ as the circle arc $\left[\xi_{0}, f^{q_{n}}\left(\xi_{0}\right)\right]$ if $n$ is even and $\left[f^{q_{n}}\left(\xi_{0}\right), \xi_{0}\right]$ if $n$ is odd. The union of two consequent fundamental segments $I_{0}^{n-1}, I_{0}^{n}$ is called the $n$th renormalization neighborhood of the point $\xi_{0}$ and we denote it by $\mathcal{V}_{n}$. Certain number of images of fundamental segments $I_{0}^{n-1}$ and $I_{0}^{n}$, under iterates of $f$, cover whole circle without overlapping beyond the endpoints and form the $n$th dynamical partition of the circle

$$
\mathbb{P}_{n}:=\mathbb{P}_{n}\left(\xi_{0}, f\right)=\left\{I_{j}^{n}:=f^{j}\left(I_{0}^{n}\right), 0 \leq j<q_{n-1}\right\} \cup\left\{I_{i}^{n-1}:=f^{i}\left(I_{0}^{n-1}\right), 0 \leq i<q_{n}\right\} .
$$

On $\mathcal{V}_{n}$ we define the Poincaré map $\pi_{n}=\left(f^{q_{n}}, f^{q_{n-1}}\right): \mathcal{V}_{n} \rightarrow \mathcal{V}_{n}$ as follows.

$$
\pi_{n}(\xi)= \begin{cases}f^{q_{n}}(\xi), & \text { if } \quad \xi \in I_{0}^{n-1} \\ f^{q_{n-1}}(\xi), & \text { if } \quad \xi \in I_{0}^{n}\end{cases}
$$

The main idea of renormalization method is to study the behaviour of the Poincare map $\pi_{n}$ as $n \rightarrow \infty$. For this, rescaling the coordinates are usually used. Let $\mathcal{A}_{n}: \mathbb{R} \rightarrow \mathbb{S}^{1}$ be an affine covering map such that $\mathcal{A}_{n}([-1,0])=I_{0}^{n}$, with $\mathcal{A}_{n}(0)=\xi_{0}$ and $\mathcal{A}_{n}(-1)=f^{q_{n}}\left(\xi_{0}\right)$. Define $a_{n} \in \mathbb{R}$ to be a positive number such that $\mathcal{A}_{n-1}\left(a_{n}\right)=f^{q_{n}}\left(\xi_{0}\right)$. Obviously, $\mathcal{A}_{n-1}$ : $\left[0, a_{n}\right] \rightarrow I_{0}^{n}$ and $\mathcal{A}_{n-1}:[-1,0] \rightarrow I_{0}^{n-1}$. Consider a mapping $\mathcal{S}_{n}:\left[-1, a_{n}\right] \rightarrow\left[-1, a_{n}\right]$ defined by $\mathcal{S}_{n}:=\left(f_{n}, g_{n}\right)=\mathcal{A}_{n-1}^{-1} \circ \pi_{n} \circ \mathcal{A}_{n-1}$, where $\mathcal{A}_{n-1}^{-1}$ is the inverse branch that 
maps $\mathcal{V}_{n}$ onto $\left[-1, a_{n}\right]$. The pair of functions $\left(f_{n}, g_{n}\right)$ is called the $n$th renormalization of $f$ with respect to $\xi_{0}$. Now we provide the exact statement of Khanin \& Vul's [28] result asserting the closeness of $\left(f_{n}, g_{n}\right)$ to the Möbius transformations $F_{n}:=F_{a_{n}, b_{n}, m_{n}}$ and $G_{n}:=G_{a_{n}, b_{n}, m_{n}, c_{n}}$ where

$$
F_{n}(z)=\frac{a_{n}+\left(a_{n}+b_{n} m_{n}\right) z}{1+\left(1-m_{n}\right) z}, \quad G_{n}(z)=\frac{-a_{n} c_{n}+\left(c_{n}-b_{n} m_{n}\right) z}{a_{n} c_{n}+\left(m_{n}-c_{n}\right) z}
$$

and

$$
c_{n}=c^{(-1)^{n}}, \quad b_{n}=\frac{\left|I_{0}^{n-1}\right|-\left|I_{q_{n-1}}^{n}\right|}{\left|I_{0}^{n-1}\right|}, \quad m_{n}=\exp \left((-1)^{n} \sum_{i=0}^{q_{n}-1} \int_{I_{i}^{n-1}} \frac{f^{\prime \prime}(x)}{2 f^{\prime}(x)} d x\right) .
$$

Theorem 1.1. [28] Let $f$ be a $C^{2+\nu}\left(\mathbb{S}^{1} \backslash\left\{\xi_{0}\right\}\right), \nu>0$ diffeomorphism with a break point $\xi_{0}$ and with irrational rotation number. Then there exist constants $C>0$ and $0<\lambda=\lambda(f)<1$ such that

$$
\left\|f_{n}-F_{n}\right\|_{C^{2}([-1,0])} \leq C \lambda^{n}, \quad\left\|g_{n}-G_{n}\right\|_{C^{1}\left(\left[0, a_{n}\right]\right)} \leq C \lambda^{n}
$$

and

$$
\left\|g_{n}^{\prime \prime}-G_{n}^{\prime \prime}\right\|_{C^{0}\left(\left[0, a_{n}\right]\right)} \leq \frac{C \lambda^{n}}{a_{n}}
$$

Moreover,

$$
\left|a_{n}+b_{n} m_{n}-c_{n}\right| \leq C a_{n} \lambda^{n} .
$$

Remark. In the case of rational rotation numbers, the renormalizations of circle diffeomorphisms with breaks was investigated by Khanin \& Vul also. They analyzed periodic trajectories of renormalization operator on one parameter family. Moreover, they showed that the Lebesgue measure of the set of parameters which correspond to the rational rotation numbers is full. Later on this result was generalized by Khmelev [20] for circle diffeomorphisms with several break points.

Remark. Recently, Cunha \& Smania 3] have studied Rauzy-Veech renormalizations of $C^{2+\nu}$-circle diffeomorphisms with several break points. The main idea of this work is to consider the piecewise-smooth circle homeomorphisms as generalized interval exchange transformations. They have proved that Rauzy-Veech renormalizations of $C^{2+\nu}$-generalized interval exchange maps satisfying a certain combinatorial conditions are approximated by Möbius transformations in $C^{2}$-topology.

\subsection{Main results}

To state our main results, we define a new class of circle diffeomorphisms with one break point. Consider the function $\mathcal{Z}_{\gamma}:[0,1) \rightarrow(0,+\infty)$, given

$$
\mathcal{Z}_{\gamma}(x)=\frac{1}{\left(\log \frac{1}{x}\right)^{\gamma}}, \quad x \in(0,1)
$$

and $\mathcal{Z}_{\gamma}(0)=0$, where $\gamma>0$. Let $f$ be a circle diffeomerphism with the break point $\xi_{0}$. Without loss of generality we may assume $\xi_{0}=0$. Denote by $\Delta^{2} f^{\prime}(\xi, \tau)$ the second symmetric difference of $f^{\prime}$, that is

$$
\Delta^{2} f^{\prime}(\xi, \tau)=f^{\prime}(\xi+\tau)+f^{\prime}(\xi-\tau)-2 f^{\prime}(\xi)
$$


where $\xi \in \mathbb{S}^{1}$ and $\tau \in\left[0, \frac{1}{2}\right]$. Suppose that there exists a constant $C>0$ such that

$$
\left\|\Delta^{2} f^{\prime}(\cdot, \tau)\right\|_{L^{\infty}\left(\mathbb{S}^{1}\right)} \leq C \tau \mathcal{Z}_{\gamma}(\tau) .
$$

Note that the class of real functions satisfying (2) with $\mathcal{Z}_{\gamma}(\tau)$ replaced by 1 , is called the Zygmund class and denoted by $\Lambda_{*}$ (see [32], p. 43). The class $\Lambda_{*}$ plays a key role to investigate trigonometric series and this class was applied to the theory of circle homeomorphisms for the first time by Jun Hu \& Sullivan (see [6], [27]). They extended the classical Denjoy's theorem to this class. Note that if $f^{\prime}$ satisfies (2) then it is not necessarily of bounded variation and vice versa. Indeed, there are examples in [24] and [32] for this statement. In this work we study the class of circle diffeomerphisms $f$ with the break point $\xi_{0}$, whose derivatives $f^{\prime}$ have bounded variation and satisfy the inequality (2). And we denote this class by $\mathrm{D}^{1+\mathcal{Z}_{\gamma}}\left(\mathbb{S}^{1} \backslash\left\{\xi_{0}\right\}\right)$. Let $f \in \mathrm{D}^{1+\mathcal{Z}_{\gamma}}\left(\mathbb{S}^{1} \backslash\left\{\xi_{0}\right\}\right)$ and its rotation number is irrational. We define two quantities $\widetilde{m}_{n}$ and $\widehat{m}_{n}$ as

$$
\widetilde{m}_{n}=\exp \left(\sum_{i=0}^{q_{n}-1} \frac{f^{\prime}\left(\xi_{i}\right)-f^{\prime}\left(\xi_{i+q_{n-1}}\right)}{2 f^{\prime}\left(\xi_{i}\right)}\right), \widehat{m}_{n}=\exp \left(\sum_{j=0}^{q_{n-1}-1} \frac{f^{\prime}\left(\xi_{j+q_{n}}\right)-f^{\prime}\left(\xi_{j}\right)}{2 f^{\prime}\left(\xi_{j+q_{n}}\right)}\right)
$$

where $\xi_{i}, \xi_{i+q_{n-1}}$ and $\xi_{j}, \xi_{j+q_{n}}$ are endpoints of the intervals $I_{i}^{n-1}, I_{j}^{n}$ respectively. Since the systems of intervals $\left\{I_{i}^{n-1}, 0 \leq i<q_{n}\right\},\left\{I_{j}^{n}, 0 \leq j<q_{n-1}\right\}$ do not intersect and $f^{\prime}$ has bounded variation, $\widetilde{m}_{n}, \widehat{m}_{n}$ are bounded for any $n \geq 1$. Below we will show that $\widetilde{m}_{n}$ and $\widehat{m}_{n}$ are exponentially close to $m_{n}$ and $c_{n} m_{n}^{-1}$ respectively, for $\gamma>1$. Using $\widetilde{m}_{n}, \widehat{m}_{n}$ we define Möbius transformations similarly as in (1) as follow

$$
\widetilde{F}_{n}(z)=\frac{a_{n}+\left(a_{n}+b_{n} \widetilde{m}_{n}\right) z}{1+\left(1-\widetilde{m}_{n}\right) z}, \quad \widehat{G}_{n}(z)=\frac{-a_{n} \widehat{m}_{n}+\left(\widehat{m}_{n}-b_{n}\right) z}{a_{n} \widehat{m}_{n}+\left(1-\widehat{m}_{n}\right) z} .
$$

Our first main result is the following.

Theorem 1.2. Let $f \in \mathrm{D}^{1+\mathcal{Z}_{\gamma}}\left(\mathbb{S}^{1} \backslash\left\{\xi_{0}\right\}\right)$ and $\gamma \in(0,1]$. Suppose the rotation number of $f$ is irrational. Then there exists a constant $C=C(f)>0$ and a natural number $N_{0}=N_{0}(f)$ such that the following inequalities

$$
\left\|f_{n}-\widetilde{F}_{n}\right\|_{C^{1}([-1,0])} \leq \frac{C}{n^{\gamma}}, \quad\left\|g_{n}-\widehat{G}_{n}\right\|_{C^{1}\left(\left[0, a_{n}\right]\right)} \leq \frac{C}{n^{\gamma}}
$$

hold for all $n \geq N_{0}$.

Note that the class $\mathrm{D}^{1+\mathcal{Z}_{\gamma}}\left(\mathbb{S}^{1} \backslash\left\{\xi_{0}\right\}\right)$ will be "better" when $\gamma$ increases. This gives more opportunities to better understand the behavior of $\mathcal{S}_{n}$. Now we consider the case $\gamma>1$. In this case, because of Theorem 2.3 stated in Section $2, f^{\prime}$ is differentiable on $\mathbb{S}^{1} \backslash\left\{\xi_{0}\right\}$, hence $\mathcal{S}_{n}^{\prime}$ is differentiable on $\left[-1, a_{n}\right] \backslash\{0\}$. Therefore we can investigate the behavior of $\mathcal{S}_{n}^{\prime \prime}$. Our second result is the following.

Theorem 1.3. Let $f \in \mathrm{D}^{1+\mathcal{Z}_{\gamma}}\left(\mathbb{S}^{1} \backslash\left\{\xi_{0}\right\}\right)$ and $\gamma>1$. Suppose the rotation number of $f$ is irrational. Then there exists a constant $C=C(f)>0$ and a natural number $N_{0}=N_{0}(f)$ such that for all $n \geq N_{0}$ the following inequalities hold

$$
\begin{gathered}
\left\|f_{n}-F_{n}\right\|_{C^{1}([-1,0])} \leq \frac{C}{n^{\gamma}}, \quad\left\|g_{n}-G_{n}\right\|_{C^{1}\left(\left[0, a_{n}\right]\right)} \leq \frac{C}{n^{\gamma}} . \\
\left\|f_{n}^{\prime \prime}-F_{n}^{\prime \prime}\right\|_{C^{0}([-1,0])} \leq \frac{C}{n^{\gamma-1}}, \quad\left\|g_{n}^{\prime \prime}-G_{n}^{\prime \prime}\right\|_{C^{0}\left(\left[0, a_{n}\right]\right)} \leq \frac{C}{a_{n} n^{\gamma-1}} .
\end{gathered}
$$


Moreover,

$$
\left|a_{n}+b_{n} m_{n}-c_{n}\right| \leq \frac{C a_{n}}{n^{\gamma}}
$$

where $F_{n}$ and $G_{n}$ are defined in (1).

\section{Remark.}

- It is obvious that the classes in Theorems 1.2 and 1.3 are wider than the class of Theorem 1.1 but the estimations are weaker.

- The Zygmund conditions is quite natural in the context of Cross-Ration Distortion (CRD). The relations between Zygmund conditions and CRD estimates have been studied in the book [24] for $\gamma=1$. Since Ratio Distortion (RD) is a partial case of $\mathrm{CRD}$, the approaches in the above book work very well to estimate RD for the considered Zygmund class. On the other hand the renormalizations can be represented by $\mathrm{RD}$, therefore we investigate the renormalizations by $\mathrm{RD}$.

The structure of paper is as follows. In Section 2, we provide brief facts about Zygmund functions and following Khanin \& Vul [28] we define a relative coordinate of an interval. Then we obtain some estimates for the distortion of interval. Moreover, we provide relations between distortion and relative coordinates of intervals. In Section 3, we get estimates for the ratio of $f^{q_{n}}$-distortion of intervals i.e., distortion of intervals with respect to $f^{q_{n}}$ for the different $\gamma^{\prime}$ 's. In Section 4, we compare the relative coordinates with Möbius transformations. Finally, in Section 5 we prove our main theorems.

\section{Ratio distortions and Zygmund condition}

\subsection{Notes on Zygmund functions}

In this subsection we provide brief facts about functions satisfying inequality (2). These facts will be used in the proof of main results. Let $I=[a, b]$ be an interval with the length less than 1 . Consider a continuous function $\mathcal{K}: I \rightarrow \mathbb{R}$. Suppose $\mathcal{K}$ satisfies the inequality (2) on $I$ i.e.,

$$
\left\|\Delta^{2} \mathcal{K}(\cdot, \tau)\right\|_{L^{\infty}([a, b])} \leq C \tau \mathcal{Z}_{\gamma}(\tau)
$$

where $\tau \in[0,|I| / 2]$. It turns out that the functions satisfying relation (8) have "a considerable degree of continuity".

Theorem 2.1. Let $\mathcal{K}: I \rightarrow \mathbb{R}$ be continuous and satisfies the inequality (8) on $I$. If $\gamma \in(0,1)$ then

$$
\omega(\delta, \mathcal{K})=\mathcal{O}\left(\delta\left(\log \frac{1}{\delta}\right)^{1-\gamma}\right)
$$

If $\gamma=1$ then

$$
\omega(\delta, \mathcal{K})=\mathcal{O}\left(\delta\left(\log \log \frac{1}{\delta}\right)\right)
$$

where $\omega(\cdot, \mathcal{K})$ is the modulus of continuity of $\mathcal{K}$.

Proof. The proof of this theorem follows closely that of [32] (p. 44) and we leave it to the reader. 
The following theorem was proved by Weiss \& Zygmund in [29]. This theorem will be used in the proof of next theorem.

Theorem 2.2. Let $\mathcal{K}: \mathbb{R} \rightarrow \mathbb{R}$ be $2 \pi$-periodic and satisfies (8) for some $\gamma \in\left(\frac{1}{2}, 1\right]$. Then $\mathcal{K}$ is absolute continuous and $\mathcal{K} \in L_{p}[0,2 \pi]$ for every $p>1$.

More direct and general proof of this theorem can be found in [7]. In this theorem the assumption $\gamma \in\left(\frac{1}{2}, 1\right]$ is crucial. The theorem is false for $\gamma \in\left(0, \frac{1}{2}\right]$. Indeed, there are functions which satisfy (8) for some $\gamma \in\left(0, \frac{1}{2}\right]$ but almost nowhere differentiable (see e.g. [32]). Next we provide a theorem on differentiability of $\mathcal{K}$ in the case of $\gamma>1$. To state this theorem we need the following function $\mathcal{P}_{\gamma}:(0,1) \rightarrow \mathbb{R}$.

$$
\mathcal{P}_{\gamma}(x)=\sum_{n=1}^{\infty} \mathcal{Z}_{\gamma}\left(x 2^{-n}\right) \text { where } x \in(0,1) \text { and } \gamma>1
$$

It is clear that $\mathcal{P}_{\gamma}$ is continuous and $\lim _{x \rightarrow 0} \mathcal{P}_{\gamma}(x)=0$.

Theorem 2.3. Let $\mathcal{K}: I \rightarrow \mathbb{R}$ be continuous and satisfies (8) for some $\gamma>1$. Then $\mathcal{K} \in C^{1}(I)$ and for any $\xi, \eta \in I$ there exists a constant $C>0$ such that

$$
\left|\mathcal{K}^{\prime}(\xi)-\mathcal{K}^{\prime}(\eta)\right| \leq C \cdot \mathcal{P}_{\gamma}(|\xi-\eta|) .
$$

Proof. We give only the sketch of proof, the details will be left to the reader. According to Theorem 2.2 the function $\mathcal{K}$ is at least absolute continuous on $I$ in the case of $\gamma>1$. Hence $\mathcal{K}^{\prime}$ exists almost everywhere and $\mathcal{K}$ is an indefinite integral of $\mathcal{K}^{\prime}$. To prove the theorem we take any points $\xi, \eta \in I$ that are Lebesgue points of $\mathcal{K}^{\prime}$ and using the same manner as in 32 (p. 44), we obtain a uniform estimate for $\left|\mathcal{K}^{\prime}(\xi)-\mathcal{K}^{\prime}(\eta)\right|$. Hence we show $\mathcal{K}^{\prime}$ is uniformly continuous and satisfies

$$
\left|\mathcal{K}^{\prime}(\xi)-\mathcal{K}^{\prime}(\eta)\right| \leq C \cdot \mathcal{P}_{\gamma}(|\xi-\eta|)
$$

on its set of Lebesgue points. Thus, it can be continuously extended to whole interval $I$.

\subsection{The distortion of interval and relative coordinate}

In this subsection we introduce the distortion of interval $I=[a, b]$ with respect to continuous and monotone function $\mathcal{K}: I \rightarrow \mathbb{R}$. We obtain some estimates for the distortion of interval. After that following Vul \& Khanin [28], we define the relative coordinate of interval and provide relations between distortion and relative coordinate. These estimates and relations will be used in the proofs of main theorems. The distortion of the interval $I$ with respect to $\mathcal{K}$ is

$$
\mathcal{R}(I ; \mathcal{K})=\frac{|\mathcal{K}(I)|}{|I|}
$$

The distortion is multiplicative with respect to composition. Henceforth, take any $x \in[a, b]$ and consider the distortions

$$
\mathcal{R}_{a}(x):=\mathcal{R}([a, x] ; \mathcal{K}) \text { and } \quad \mathcal{R}_{b}(x):=\mathcal{R}([x, b] ; \mathcal{K})
$$


Below we study the distortions $\mathcal{R}_{a}(x)$ and $\mathcal{R}_{b}(x)$ as the functions of $x \in[a, b]$. Consider the following function $\Omega:(0,1) \times(0,+\infty) \rightarrow \mathbb{R}$,

$$
\Omega(\delta, \gamma)= \begin{cases}\delta\left(\log \frac{1}{\delta}\right)^{1-\gamma} & \text { if }(\delta, \gamma) \in(0,1) \times(0,1) \\ \delta\left(\log \log \frac{1}{\delta}\right) & \text { if }(\delta, \gamma) \in(0,1) \times\{1\} \\ \delta & \text { if }(\delta, \gamma) \in(0,1) \times(1,+\infty)\end{cases}
$$

In fact the function $\Omega(\delta, \gamma)$ is the modulus of continuity of the functions satisfying relation (8) for the different cases of $\gamma$.

Denote by $\mathrm{D}^{1+\mathcal{Z}_{\gamma}}(I)$ the class of diffeomorphisms $\mathcal{K}$ whose derivatives $\mathcal{K}^{\prime}$ satisfy the inequality (8) on $I$. In the sequel we prove several lemmas which will be used in the proofs of main theorems.

Lemma 2.4. Let $\mathcal{K} \in \mathrm{D}^{1+\mathcal{Z}_{\gamma}}(I)$ and $\gamma \in(0,+\infty)$. Then we have

$$
\frac{\mathcal{R}_{a}(x)}{\mathcal{R}_{b}(x)}-1=\frac{\mathcal{K}^{\prime}(a)-\mathcal{K}^{\prime}(b)}{2 \mathcal{K}^{\prime}(b)}+\mathcal{O}\left(|I| \cdot \mathcal{Z}_{\gamma}(|I|)+\left|\mathcal{K}^{\prime}(a)-\mathcal{K}^{\prime}(b)\right| \cdot \Omega(|I|, \gamma)\right) .
$$

Proof. Since $\mathcal{K}^{\prime}$ satisfies (8), similarly as in [24] (p. 293) we have

$$
\mathcal{R}_{a}(x)=\frac{\mathcal{K}^{\prime}(x)+\mathcal{K}^{\prime}(a)}{2}+\mathcal{O}\left(|I| \cdot \mathcal{Z}_{\gamma}(|I|)\right), \quad \mathcal{R}_{b}(x)=\frac{\mathcal{K}^{\prime}(b)+\mathcal{K}^{\prime}(x)}{2}+\mathcal{O}\left(|I| \cdot \mathcal{Z}_{\gamma}(|I|)\right) .
$$

The last two relations imply

$$
\frac{\mathcal{R}_{a}(x)}{\mathcal{R}_{b}(x)}-1=\frac{\mathcal{K}^{\prime}(a)-\mathcal{K}^{\prime}(b)}{\mathcal{K}^{\prime}(b)+\mathcal{K}^{\prime}(x)}+\mathcal{O}\left(|I| \cdot \mathcal{Z}_{\gamma}(|I|)\right) .
$$

It is obvious

$$
\frac{\mathcal{K}^{\prime}(a)-\mathcal{K}^{\prime}(b)}{\mathcal{K}^{\prime}(b)+\mathcal{K}^{\prime}(x)}=\frac{\mathcal{K}^{\prime}(a)-\mathcal{K}^{\prime}(b)}{2 \mathcal{K}^{\prime}(b)}+\left|\mathcal{K}^{\prime}(a)-\mathcal{K}^{\prime}(b)\right| \mathcal{O}\left(\left|\mathcal{K}^{\prime}(x)-\mathcal{K}^{\prime}(b)\right|\right) .
$$

Hence, the claim of lemma follows from Theorems 2.1, 2.3 and above relations.

Note that according to Theorem 2.3 the function $\mathcal{K}^{\prime}$ is differentiable in the case of $\gamma>1$. Hence we have the following.

Corollary 2.5. Let $\mathcal{K} \in \mathrm{D}^{1+\mathcal{Z}_{\gamma}}(I)$ for some $\gamma \in(1,+\infty)$. Then we have

$$
\frac{\mathcal{R}_{a}(x)}{\mathcal{R}_{b}(x)}-1=-\int_{a}^{b} \frac{\mathcal{K}^{\prime \prime}(y)}{2 \mathcal{K}^{\prime}(y)} d y+\mathcal{O}\left(|I| \cdot \mathcal{Z}_{\gamma}(|I|)+\left|\mathcal{K}^{\prime}(a)-\mathcal{K}^{\prime}(b)\right| \cdot \Omega(|I|, \gamma)\right) .
$$

Now we define the relative coordinate $z: I \rightarrow[0,1]$ as follows

$$
z=\frac{b-x}{b-a} \text {. }
$$

Next we prove the following.

Lemma 2.6. Let $\mathcal{K} \in \mathrm{D}^{1+\mathcal{Z}_{\gamma}}(I)$ and $\gamma \in(0,+\infty)$. Then we have

$$
(x-a)(b-x)\left(\frac{\mathcal{R}_{b}^{\prime}(x)-\mathcal{R}_{a}^{\prime}(x)}{b-a}\right)=\frac{1}{2}\left(z \mathcal{K}^{\prime}(a)+(1-z) \mathcal{K}^{\prime}(b)-\mathcal{K}^{\prime}(x)\right)+\mathcal{O}\left(|I| \cdot \mathcal{Z}_{\gamma}(|I|)\right)
$$


Proof. By differentiating $\mathcal{R}_{a}$ and $\mathcal{R}_{b}$ we obtain

$$
\mathcal{R}_{a}^{\prime}(x)=\frac{\mathcal{K}^{\prime}(x)-\mathcal{R}_{a}(x)}{x-a} \quad \text { and } \quad \mathcal{R}_{b}^{\prime}(x)=\frac{\mathcal{R}_{b}(x)-\mathcal{K}^{\prime}(x)}{b-x} .
$$

Applying the same arguments as in the proof of Lemma 2.4, we can show that

$\mathcal{R}_{a}^{\prime}(x)=\frac{1}{2} \cdot \frac{\mathcal{K}^{\prime}(x)-\mathcal{K}^{\prime}(a)}{x-a}+\frac{1}{1-z} \mathcal{O}\left(\mathcal{Z}_{\gamma}(|I|)\right), \quad \mathcal{R}_{b}^{\prime}(x)=\frac{1}{2} \cdot \frac{\mathcal{K}^{\prime}(b)-\mathcal{K}^{\prime}(x)}{b-x}+\frac{1}{z} \mathcal{O}\left(\mathcal{Z}_{\gamma}(|I|)\right)$.

Hence

$$
(x-a)(b-x)\left(\frac{\mathcal{R}_{b}^{\prime}(x)-\mathcal{R}_{a}^{\prime}(x)}{b-a}\right)=\frac{1}{2}\left(z \mathcal{K}^{\prime}(a)+(1-z) \mathcal{K}^{\prime}(b)-\mathcal{K}^{\prime}(x)\right)+\mathcal{O}\left(|I| \cdot \mathcal{Z}_{\gamma}(|I|)\right) .
$$

Lemma 2.6 is proved.

Next we estimate the expression in the right hand side of equation (11). For this we define the following function $T_{\gamma}:[0,1 / 2] \times(0,1) \rightarrow \mathbb{R}$ as

$$
T_{\gamma}(s, t)=s \int_{s}^{1} \frac{\mathcal{Z}_{\gamma}(x t) d x}{x}+\int_{0}^{s} \mathcal{Z}_{\gamma}(x t) d x \text { if } s \in(0,1 / 2]
$$

and $T_{\gamma}(0, t)=0$, for any $t \in(0,1)$.

Lemma 2.7. Let $\mathcal{K} \in \mathrm{D}^{1+\mathcal{Z}_{\gamma}}(I)$ and $\gamma \in(0,+\infty)$. Then there exists a constant $C>0$ such that

$$
\left|z \mathcal{K}^{\prime}(a)+(1-z) \mathcal{K}^{\prime}(b)-\mathcal{K}^{\prime}(x)\right| \leq C\left\{\begin{array}{l}
|I| \cdot T_{\gamma}(z,|I|) \quad \text { if } z \in\left[0, \frac{1}{2}\right] \\
|I| \cdot T_{\gamma}(1-z,|I|) \quad \text { if } z \in\left(\frac{1}{2}, 1\right] .
\end{array}\right.
$$

Proof. Let us consider the function $\kappa(z):=\mathcal{K}^{\prime}(b+z(a-b)), z \in[0,1]$. It is clear that, to prove the lemma we have to estimate $|(1-z) \kappa(0)+z \kappa(1)-\kappa(z)|$. Note that, since $\mathcal{K}^{\prime}$ satisfies (8) we have

$$
\left|\frac{1}{2} \kappa(\xi+\tau)+\frac{1}{2} \kappa(\xi-\tau)-\kappa(\xi)\right| \leq C \cdot|\tau||I| \mathcal{Z}_{\gamma}(|\tau||I|)
$$

for all $\xi+\tau, \xi-\tau \in[0,1]$. Denote by $\mathcal{D}_{\ell}, \ell=0,1,2, \ldots$ the dyadic partition of $[0,1]$. Take any $z \in(0,1)$, fix it and denote by $J_{\ell}=\left[\mathfrak{a}_{\ell}, \mathfrak{b}_{\ell}\right)$ the dyadic interval of $\mathcal{D}_{\ell}$ which contains $z$. Using this interval we define the following quantities

$$
\mathfrak{r}_{\ell}=\frac{\mathfrak{b}_{\ell}-z}{\mathfrak{b}_{\ell}-\mathfrak{a}_{\ell}} \kappa\left(\mathfrak{a}_{\ell}\right)+\frac{z-\mathfrak{a}_{\ell}}{\mathfrak{b}_{\ell}-\mathfrak{a}_{\ell}} \kappa\left(\mathfrak{b}_{\ell}\right)-\kappa(z), \quad \mathfrak{t}_{\ell}=\frac{1}{2} \kappa\left(\mathfrak{a}_{\ell}\right)+\frac{1}{2} \kappa\left(\mathfrak{b}_{\ell}\right)-\kappa\left(\frac{\mathfrak{a}_{\ell}+\mathfrak{b}_{\ell}}{2}\right) .
$$

After an easy computation we get

$$
\mathfrak{r}_{\ell+1}-\mathfrak{r}_{\ell}=-\frac{\operatorname{dist}\left(z, \partial\left(J_{\ell}\right)\right)}{\left|J_{\ell+1}\right|} \mathfrak{t}_{\ell}
$$

Using this equality we obtain

$$
\left|\mathfrak{r}_{\mathfrak{o}}\right| \leq \sum_{\ell=0}^{\infty} \frac{\operatorname{dist}\left(z, \partial\left(J_{\ell}\right)\right)}{\left|J_{\ell+1}\right|}\left|\mathfrak{t}_{\ell}\right|
$$


There are two possibilities for $z$ : either $z \in\left(0, \frac{1}{2}\right]$ or $z \in\left(\frac{1}{2}, 1\right)$. Consider the first case. Let $m$ be the biggest natural number such that $z \leq 2^{-m}$. It is easy to verify that

$$
\operatorname{dist}\left(z, \partial\left(J_{\ell}\right)\right) \leq \begin{cases}z, & \text { if } \ell \leq m \\ \left|J_{\ell}\right|, & \text { if } \ell>m\end{cases}
$$

Similarly, in the second case we define the biggest natural number $p$ such that $1-z \leq 2^{-p}$. One can verify that

$$
\operatorname{dist}\left(z, \partial\left(J_{\ell}\right)\right) \leq \begin{cases}1-z, & \text { if } \ell \leq p \\ \left|J_{\ell}\right|, & \text { if } \ell>p\end{cases}
$$

Consequently,

$$
\left|\mathfrak{r}_{\mathfrak{o}}\right| \leq \begin{cases}z \sum_{\ell=0}^{m} 2^{\ell+1}\left|\mathfrak{t}_{\ell}\right|+2 \sum_{\ell=m+1}^{\infty}\left|\mathfrak{t}_{\ell}\right|, & \text { if } \quad z \in\left(0, \frac{1}{2}\right] \\ (1-z) \sum_{\ell=0}^{p} 2^{\ell+1}\left|\mathfrak{t}_{\ell}\right|+2 \sum_{\ell=p+1}^{\infty}\left|\mathfrak{t}_{\ell}\right|, & \text { if } \quad z \in\left(\frac{1}{2}, 1\right) .\end{cases}
$$

Using inequality (12) we get

$$
\left|\mathfrak{r}_{\mathfrak{o}}\right| \leq C \begin{cases}|I|\left(z \sum_{\ell=0}^{m} \mathcal{Z}_{\gamma}\left(2^{-\ell-1}|I|\right)+\sum_{\ell=m+1}^{\infty} 2^{-\ell} \mathcal{Z}_{\gamma}\left(2^{-\ell-1}|I|\right)\right), & \text { if } \quad z \in\left(0, \frac{1}{2}\right] \\ |I|\left((1-z) \sum_{\ell=0}^{p} \mathcal{Z}_{\gamma}\left(2^{-\ell-1}|I|\right)+\sum_{\ell=p+1}^{\infty} 2^{-\ell} \mathcal{Z}_{\gamma}\left(2^{-\ell-1}|I|\right)\right), & \text { if } \quad z \in\left(\frac{1}{2}, 1\right) .\end{cases}
$$

It is obvious that if $z \in\left(0, \frac{1}{2}\right]$ then

$$
z \sum_{\ell=0}^{m} \mathcal{Z}_{\gamma}\left(2^{-\ell-1}|I|\right)+\sum_{\ell=m+1}^{\infty} 2^{-\ell} \mathcal{Z}_{\gamma}\left(2^{-\ell-1}|I| \leq C \cdot T(z,|I|) .\right.
$$

Similarly, if $z \in\left(\frac{1}{2}, 1\right)$ then

$$
(1-z) \sum_{\ell=0}^{p} \mathcal{Z}_{\gamma}\left(2^{-\ell-1}|I|\right)+\sum_{\ell=p+1}^{\infty} 2^{-\ell} \mathcal{Z}_{\gamma}\left(2^{-\ell-1}|I| \leq C \cdot T(1-z,|I|) .\right.
$$

Thus

$$
\left|\mathfrak{r}_{\mathfrak{o}}\right| \leq C \begin{cases}|I| \cdot T_{\gamma}(z,|I|), & \text { if } \quad z \in\left(0, \frac{1}{2}\right] \\ |I| \cdot T_{\gamma}(1-z,|I|), & \text { if } \quad z \in\left(\frac{1}{2}, 1\right) .\end{cases}
$$

On the other hand

$$
|(1-z) \kappa(0)+z \kappa(1)-\kappa(z)|=\left|\mathfrak{r}_{\mathfrak{o}}\right| .
$$

Hence we have proved Lemma 2.7 for $z \in(0,1)$. For $z \in\{0,1\}$ the claim of lemma is obvious.

It is easy to see that the function $T_{\gamma}(z,|I|)$ is increasing function of $z$ on $\left[0, \frac{1}{2}\right]$. Hence the function $T_{\gamma}(1-z,|I|)$ is decreasing function of $z$ on $\left[\frac{1}{2}, 1\right]$. Therefore $T_{\gamma}(z,|I|) \leq T_{\gamma}\left(\frac{1}{2},|I|\right)$ for all $z \in\left[0, \frac{1}{2}\right]$ and $T_{\gamma}(1-z,|I|) \leq T\left(\frac{1}{2},|I|\right)$ for all $z \in\left[\frac{1}{2}, 1\right]$. Moreover, if the length of interval $I$ is sufficiently small then it can be easily shown that

$$
T_{\gamma}\left(\frac{1}{2},|I|\right)=\mathcal{O}\left(\mathcal{Z}_{\gamma}(|I|)\right) .
$$

Thus Lemmas 2.6 and 2.7 imply the following. 
Corollary 2.8. Let $\mathcal{K} \in \mathrm{D}^{1+\mathcal{Z}_{\gamma}}(I)$ and $\gamma \in(0,+\infty)$. If the length of interval $I$ is sufficiently small then we have

$$
(x-a)(b-x)\left|\frac{\mathcal{R}_{b}^{\prime}(x)-\mathcal{R}_{a}^{\prime}(x)}{b-a}\right|=\mathcal{O}\left(|I| \mathcal{Z}_{\gamma}(|I|)\right) .
$$

Next we consider the subcase $\gamma \in(1,+\infty)$. Due to Theorem 2.3, $\mathcal{K}^{\prime}$ is differentiable. Therefore $\mathcal{R}_{a}^{\prime}$ and $\mathcal{R}_{b}^{\prime}$ are differentiable and we have the following.

Lemma 2.9. Let $\mathcal{K} \in \mathrm{D}^{1+\mathcal{Z}_{\gamma}}(I)$ and $\gamma \in(1,+\infty)$. Then there exists a constant $C>0$ such that

$$
\begin{gathered}
\left|(x-a)(b-x)\left(\mathcal{R}_{a}^{\prime \prime}(x)-\mathcal{R}_{b}^{\prime \prime}(x)\right)\right| \leq C \cdot|I| \mathcal{P}_{\gamma}(|I|), \\
\left|\mathcal{R}_{b}^{\prime}(x)-\mathcal{R}_{a}^{\prime}(x)\right| \leq C \cdot \mathcal{P}_{\gamma}(|I|)
\end{gathered}
$$

where function $\mathcal{P}_{\gamma}$ is defined in (9).

Proof. The proof lemma follows from easy computations and Theorem 2.3 .

\section{Estimates for the ratio of $f^{q_{n}}$-distortions}

In this section we first define relative coordinates on the intervals of dynamical partition $\mathbb{P}_{n}$ and the ratio of $f^{q_{n}}$-distortions i.e., distortions of intervals with respect to $f^{q_{n}}$. Then we describe the ratio of $f^{q_{n}}$-distortions by initial relative coordinates and provide estimates for this description and its derivatives. Note that the relative coordinates on intervals of dynamical partition $\mathbb{P}_{n}$ was introduced and very well investigated by Sinai \& Khanin in the work [14. Here and in the next sections we always assume the rotation number is irrational and we use in the following formulations: $b-a$ where $a, b \in \mathbb{S}^{1}$ and often the map $f: \mathbb{S}^{1} \rightarrow \mathbb{S}^{1}$ with $f(b)-f(a)$, even if correctly these had to be replaced by the lifts $\tilde{b}-\tilde{a}$ where $\tilde{a}, \tilde{b} \in[0,1)$ and $F: \mathbb{R} \rightarrow \mathbb{R}$ with $F(\tilde{b})-F(\tilde{a})$ respectively. Having these in mind we introduce the relative coordinates $z_{i}: I_{i}^{n-1} \rightarrow[0,1]$ for all $0 \leq i \leq q_{n}$ and $\bar{z}_{j}: I_{j}^{n} \rightarrow[0,1]$ for all $0 \leq j \leq q_{n-1}$, by the formulae respectively:

$$
z_{i}=\frac{\xi_{i}-x}{\xi_{i}-\xi_{i+q_{n-1}}}, \quad x \in I_{i}^{n-1} \quad \text { and } \quad \bar{z}_{j}=\frac{\xi_{j+q_{n}}-y}{\xi_{j+q_{n}}-\xi_{j}}, \quad y \in I_{j}^{n} .
$$

Here and in the following, we discuss only the case where $n$ is even, the case where $n$ is odd is obtained by reversing the orientation of $\mathbb{S}^{1}$. Next we define

$$
\begin{aligned}
& \widetilde{\Upsilon}_{n}(x)=\log \frac{\mathcal{R}\left(\left[\xi_{q_{n-1}}, x\right] ; f^{q_{n}}\right)}{\mathcal{R}\left(\left[x, \xi_{0}\right] ; f^{q_{n}}\right)}+\log \widetilde{m}_{n}, \quad x \in I_{0}^{n-1} . \\
& \widehat{\Upsilon}_{n}(x)=\log \frac{\mathcal{R}\left(\left[\xi_{0}, x\right] ; f^{q_{n-1}}\right)}{\mathcal{R}\left(\left[x, \xi_{q_{n}}\right] ; f^{q_{n-1}}\right)}+\log \widehat{m}_{n}, \quad x \in I_{0}^{n} .
\end{aligned}
$$

To be easy to write we use the following notations

$$
\begin{gathered}
\alpha_{i}:=\xi_{i+q_{n-1}}, \quad \beta_{i}:=\xi_{i} \quad \text { and } \quad x_{i}:=f^{i}(x) \in I_{i}^{n-1}, \quad 0 \leq i \leq q_{n} . \\
\bar{\alpha}_{j}:=\xi_{j}, \quad \bar{\beta}_{j}:=\xi_{j+q_{n}} \quad \text { and } \quad y_{j}:=f^{j}(y) \in I_{j}^{n}, \quad 0 \leq j \leq q_{n-1} .
\end{gathered}
$$


Hence

$$
x=\beta_{0}+z_{0}\left(\alpha_{0}-\beta_{0}\right) \quad \text { and } \quad y=\bar{\beta}_{0}+\bar{z}_{0}\left(\bar{\alpha}_{0}-\bar{\beta}_{0}\right) .
$$

So, we set

$$
\widetilde{\Upsilon}_{n}\left(z_{0}\right):=\widetilde{\Upsilon}_{n}\left(\beta_{0}+z_{0}\left(\alpha_{0}-\beta_{0}\right)\right), \quad \widehat{\Upsilon}_{n}\left(\bar{z}_{0}\right):=\widehat{\Upsilon}_{n}\left(\bar{\beta}_{0}+\bar{z}_{0}\left(\bar{\alpha}_{0}-\bar{\beta}_{0}\right)\right) .
$$

Below we estimate $\widetilde{\Upsilon}_{n}$ and $\widehat{\Upsilon}_{n}$. These estimates will be used in the next section to approximate relative coordinates with Möbius transformations.

Lemma 3.1. Let $f \in \mathrm{D}^{1+\mathcal{Z}_{\gamma}}\left(\mathbb{S}^{1} \backslash\left\{\xi_{0}\right\}\right)$ and $\gamma \in(0,+\infty)$. Then there exists a constant $C>0$ such that

$$
\max _{z_{0} \in[0,1]}\left|\widetilde{\Upsilon}_{n}\left(z_{0}\right)\right| \leq \frac{C}{n^{\gamma}}, \quad \max _{z_{0} \in[0,1]}\left|\widehat{\Upsilon}_{n}\left(\bar{z}_{0}\right)\right| \leq \frac{C}{n^{\gamma}}
$$

for all $n \geq 1$.

Proof. We prove only first inequality, the second inequality can be proved analogously. Since, the ratio distortion is multiplicative with respect to composition and using notation (10) we have

$$
\widetilde{\Upsilon}_{n}\left(z_{0}\right)=\sum_{i=0}^{q_{n}-1} \log \frac{\mathcal{R}_{\alpha_{i}}\left(x_{i}\right)}{\mathcal{R}_{\beta_{i}}\left(x_{i}\right)}+\log \widetilde{m}_{n}
$$

Because the sytem of intervals $\left\{I_{i}^{n-1}=\left[\alpha_{i}, \beta_{i}\right], 0 \leq i<q_{n}\right\}$ do not contain the break point, utilizing Lemma 2.4 we get

$$
\begin{gathered}
\sum_{i=0}^{q_{n}-1} \log \frac{\mathcal{R}_{\alpha_{i}}\left(x_{i}\right)}{\mathcal{R}_{\beta_{i}}\left(x_{i}\right)}=\sum_{i=0}^{q_{n}-1} \log \left(1+\frac{f^{\prime}\left(\alpha_{i}\right)-f^{\prime}\left(\beta_{i}\right)}{2 f^{\prime}\left(\beta_{i}\right)}\right)+ \\
\mathcal{O}\left(\sum_{i=0}^{q_{n}-1}\left|I_{i}^{n-1}\right| \cdot \mathcal{Z}_{\gamma}\left(\left|I_{i}^{n-1}\right|\right)+\left|f^{\prime}\left(\alpha_{i}\right)-f^{\prime}\left(\beta_{i}\right)\right| \cdot \Omega\left(\left|I_{i}^{n-1}\right|, \gamma\right)\right) .
\end{gathered}
$$

It is clear

$$
\sum_{i=0}^{q_{n}-1}\left|I_{i}^{n-1}\right| \mathcal{Z}_{\gamma}\left(\left|I_{i}^{n-1}\right|\right)=\mathcal{O}\left(\mathcal{Z}_{\gamma}\left(d_{n-1}\right)\right)
$$

and

$$
\sum_{i=0}^{q_{n}-1}\left|f^{\prime}\left(\alpha_{i}\right)-f^{\prime}\left(\beta_{i}\right)\right| \cdot \Omega\left(\left|I_{i}^{n-1}\right|, \gamma\right)=\mathcal{O}\left(\Omega\left(d_{n-1}, \gamma\right)\right)
$$

where $d_{n}:=\left\|f^{q_{n}}-\mathrm{Id}\right\|_{C^{0}}$. It is well known that $d_{n}=\mathcal{O}\left(\lambda^{n}\right)$ (see [4]) and this implies

$$
\mathcal{Z}_{\gamma}\left(d_{n-1}\right)+\Omega\left(d_{n-1}, \gamma\right)=\mathcal{O}\left(\frac{1}{n^{\gamma}}\right) .
$$

Therefore, using Taylor's formula and combining (18)-(20) we get

$$
\sum_{i=0}^{q_{n}-1} \log \frac{\mathcal{R}_{\alpha_{i}}\left(x_{i}\right)}{\mathcal{R}_{\beta_{i}}\left(x_{i}\right)}=-\log \widetilde{m}_{n}+\mathcal{O}\left(\frac{1}{n^{\gamma}}\right) .
$$


Thus and so

$$
\max _{z_{0} \in[0,1]}\left|\widetilde{\Upsilon}_{n}\left(z_{0}\right)\right| \leq \frac{C}{n^{\gamma}}
$$

Lemma 3.1 is proved.

The following estimates will be used in the next section to approximate relative coordinates with Möbius transformations in $C^{1}$-topology.

Lemma 3.2. Let $f \in \mathrm{D}^{1+\mathcal{Z}_{\gamma}}\left(\mathbb{S}^{1} \backslash\left\{\xi_{0}\right\}\right)$ and $\gamma \in(0,+\infty)$. Then there exists a constant $C>0$ and a natural number $N_{0}=N_{0}(f)$ such that

$$
\max _{z_{0} \in[0,1]}\left|z_{0}\left(1-z_{0}\right) \frac{\mathrm{d} \widetilde{\Upsilon}_{n}\left(z_{0}\right)}{\mathrm{d} z_{0}}\right| \leq \frac{C}{n^{\gamma}}, \quad \max _{\bar{z}_{0} \in[0,1]}\left|\bar{z}_{0}\left(1-\bar{z}_{0}\right) \frac{\mathrm{d} \widehat{\Upsilon}_{n}\left(\bar{z}_{0}\right)}{\mathrm{d} \bar{z}_{0}}\right| \leq \frac{C}{n^{\gamma}}
$$

for all $n \geq N_{0}$.

Proof. We prove the first inequality, the second one can be proved analogously. Setting

$$
\Psi\left(x_{i}\right):=\log \frac{\mathcal{R}_{\alpha_{i}}\left(x_{i}\right)}{\mathcal{R}_{\beta_{i}}\left(x_{i}\right)}
$$

rewrite $\widetilde{\Upsilon}_{n}$ as follows

$$
\widetilde{\Upsilon}_{n}\left(z_{0}\right)=\sum_{i=0}^{q_{n}-1} \Psi\left(x_{i}\right)+\log \widetilde{m}_{n}
$$

It is clear

$$
\frac{\mathrm{d} \widetilde{\Upsilon}_{n}\left(z_{0}\right)}{\mathrm{d} z_{0}}=\frac{\mathrm{d} \widetilde{\Upsilon}_{n}(x)}{\mathrm{d} x} \cdot \frac{\mathrm{d} x}{\mathrm{~d} z_{0}}=\left(\alpha_{0}-\beta_{0}\right) \cdot \frac{\mathrm{d} \widetilde{\Upsilon}_{n}(x)}{\mathrm{d} x}
$$

and

$$
\frac{\mathrm{d} \Psi\left(x_{i}\right)}{\mathrm{d} x}=\frac{\mathrm{d} \Psi\left(x_{i}\right)}{\mathrm{d} x_{i}} \cdot \frac{\mathrm{d} x_{i}}{\mathrm{~d} x}=\frac{\mathrm{d} \Psi\left(x_{i}\right)}{\mathrm{d} x_{i}} \cdot\left(f^{i}(x)\right)^{\prime} .
$$

Using Finzi's inequality (see [1] for finitely many breaks) we obtain

$$
e^{-\nu} \leq \frac{\left(f^{i}(x)\right)^{\prime}\left(\alpha_{0}-\beta_{0}\right)}{\left(\alpha_{i}-\beta_{i}\right)} \leq e^{\nu} \quad \text { and } \quad e^{-2 \nu} \leq \frac{z_{0}\left(1-z_{0}\right)}{z_{i}\left(1-z_{i}\right)} \leq e^{2 \nu} .
$$

From (22) $-(25)$ we get

$$
\left|z_{0}\left(1-z_{0}\right) \frac{\mathrm{d} \widetilde{\Upsilon}_{n}\left(z_{0}\right)}{\mathrm{d} z_{0}}\right| \leq e^{3 \nu}\left|\sum_{i=0}^{q_{n}-1} z_{i}\left(1-z_{i}\right)\left(\alpha_{i}-\beta_{i}\right) \frac{\mathrm{d} \Psi\left(x_{i}\right)}{\mathrm{d} x_{i}}\right| .
$$

By differentiating (21) we have

$$
\begin{gathered}
\frac{\mathrm{d} \Psi\left(x_{i}\right)}{\mathrm{d} x_{i}}=\frac{1}{\mathcal{R}_{\alpha_{i}}\left(x_{i}\right)} \cdot \frac{\mathrm{d} \mathcal{R}_{\alpha_{i}}\left(x_{i}\right)}{\mathrm{d} x_{i}}-\frac{1}{\mathcal{R}_{\beta_{i}}\left(x_{i}\right)} \cdot \frac{\mathrm{d} \mathcal{R}_{\beta_{i}}\left(x_{i}\right)}{\mathrm{d} x_{i}}= \\
\left(\frac{1}{\mathcal{R}_{\alpha_{i}}\left(x_{i}\right)}-\frac{1}{\mathcal{R}_{\beta_{i}}\left(x_{i}\right)}\right) \cdot \frac{\mathrm{d} \mathcal{R}_{\alpha_{i}}\left(x_{i}\right)}{\mathrm{d} x_{i}}+\frac{1}{\mathcal{R}_{\beta_{i}}\left(x_{i}\right)} \cdot\left(\frac{\mathrm{d} \mathcal{R}_{\alpha_{i}}\left(x_{i}\right)}{\mathrm{d} x_{i}}-\frac{\mathrm{d} \mathcal{R}_{\beta_{i}}\left(x_{i}\right)}{\mathrm{d} x_{i}}\right) .
\end{gathered}
$$


Utilizing mean value theorem and Theorems 2.1 , 2.3 we get

$$
\left|\frac{1}{\mathcal{R}_{\alpha_{i}}\left(x_{i}\right)}-\frac{1}{\mathcal{R}_{\beta_{i}}\left(x_{i}\right)}\right|=\left|\frac{1}{f^{\prime}\left(\check{\alpha}_{i}\right)}-\frac{1}{f^{\prime}\left(\check{\beta}_{i}\right)}\right| \leq C \cdot \Omega\left(d_{n-1}, \gamma\right)
$$

for any $\gamma \in(0,+\infty)$, where $\check{\alpha}_{i} \in\left[\alpha_{i}, x_{i}\right]$ and $\check{\beta}_{i} \in\left[x_{i}, \beta_{i}\right]$. Using this, we estimate $\left|\frac{\mathrm{d} \Psi\left(x_{i}\right)}{\mathrm{d} x_{i}}\right|$ as

$$
\left|\frac{\mathrm{d} \Psi\left(x_{i}\right)}{\mathrm{d} x_{i}}\right| \leq C \cdot \Omega\left(d_{n-1}, \gamma\right)\left|\frac{\mathrm{d} \mathcal{R}_{\alpha_{i}}\left(x_{i}\right)}{\mathrm{d} x_{i}}\right|+\frac{1}{\inf _{\mathbb{S}^{1}} f^{\prime}(\xi)} \cdot\left|\frac{\mathrm{d} \mathcal{R}_{\alpha_{i}}\left(x_{i}\right)}{\mathrm{d} x_{i}}-\frac{\mathrm{d} \mathcal{R}_{\beta_{i}}\left(x_{i}\right)}{\mathrm{d} x_{i}}\right| .
$$

Applying this inequality to the right hand side of (26) we get

$$
\begin{gathered}
\left|z_{0}\left(1-z_{0}\right) \frac{\mathrm{d} \widetilde{\Upsilon}_{n}\left(z_{0}\right)}{\mathrm{d} z_{0}}\right| \leq C e^{3 \nu} \Omega\left(d_{n-1}, \gamma\right) \sum_{i=0}^{q_{n}-1} z_{i}\left(1-z_{i}\right)\left|\alpha_{i}-\beta_{i}\right|\left|\frac{\mathrm{d} \mathcal{R}_{\alpha_{i}}\left(x_{i}\right)}{\mathrm{d} x_{i}}\right| \\
+\frac{e^{3 \nu}}{\inf _{\mathbb{S}^{1}} f^{\prime}(\xi)} \sum_{i=0}^{q_{n}-1} z_{i}\left(1-z_{i}\right)\left|\alpha_{i}-\beta_{i}\right|\left|\frac{\mathrm{d} \mathcal{R}_{\alpha_{i}}\left(x_{i}\right)}{\mathrm{d} x_{i}}-\frac{\mathrm{d} \mathcal{R}_{\beta_{i}}\left(x_{i}\right)}{\mathrm{d} x_{i}}\right|:=A_{n}+B_{n} .
\end{gathered}
$$

Next we estimate $A_{n}$ and $B_{n}$. To estimate $A_{n}$ we use the following obvious equality

$$
z_{i}\left(1-z_{i}\right)\left|\alpha_{i}-\beta_{i}\right|\left|\frac{\mathrm{d} \mathcal{R}_{\alpha_{i}}\left(x_{i}\right)}{\mathrm{d} x_{i}}\right|=z_{i}\left|f^{\prime}\left(x_{i}\right)-f^{\prime}\left(\check{\alpha}_{i}\right)\right| .
$$

Therefore

$$
A_{n}=C e^{3 \nu} \Omega\left(d_{n-1}, \gamma\right) \sum_{i=0}^{q_{n}-1} z_{i}\left|f^{\prime}\left(x_{i}\right)-f^{\prime}\left(\check{\alpha}_{i}\right)\right| .
$$

Since $f^{\prime}$ has bounded variation and the system of intervals $\left\{\left[x_{i}, \check{\alpha}_{i}\right], \quad 0 \leq i<q_{n}\right\}$ do not intersect, we have

$$
A_{n}=\mathcal{O}\left(\Omega\left(d_{n-1}, \gamma\right)\right)
$$

Next we estimate $B_{n}$. By definition of relative coordinate $z_{i}$ we have

$$
z_{i}\left(1-z_{i}\right)\left|\alpha_{i}-\beta_{i}\right|\left|\frac{\mathrm{d} \mathcal{R}_{\alpha_{i}}\left(x_{i}\right)}{\mathrm{d} x_{i}}-\frac{\mathrm{d} \mathcal{R}_{\beta_{i}}\left(x_{i}\right)}{\mathrm{d} x_{i}}\right|=\frac{\left(x_{i}-\alpha_{i}\right)\left(\beta_{i}-x_{i}\right)}{\beta_{i}-\alpha_{i}}\left|\frac{\mathrm{d} \mathcal{R}_{\alpha_{i}}\left(x_{i}\right)}{\mathrm{d} x_{i}}-\frac{\mathrm{d} \mathcal{R}_{\beta_{i}}\left(x_{i}\right)}{\mathrm{d} x_{i}}\right| .
$$

Due to Corollary 2.8 we get

$$
\frac{\left(x_{i}-\alpha_{i}\right)\left(\beta_{i}-x_{i}\right)}{\beta_{i}-\alpha_{i}}\left|\frac{\mathrm{d} \mathcal{R}_{\alpha_{i}}\left(x_{i}\right)}{\mathrm{d} x_{i}}-\frac{\mathrm{d} \mathcal{R}_{\beta_{i}}\left(x_{i}\right)}{\mathrm{d} x_{i}}\right|=\mathcal{O}\left(\left|I_{i}^{n-1}\right| \mathcal{Z}_{\gamma}\left(\left|I_{i}^{n-1}\right|\right)\right)
$$

for sufficiently lagre $n$. Hence

$$
B_{n}=\mathcal{O}\left(\sum_{i=0}^{q_{n}-1}\left|I_{i}^{n-1}\right| \mathcal{Z}_{\gamma}\left(\left|I_{i}^{n-1}\right|\right)\right)=\mathcal{O}\left(\mathcal{Z}_{\gamma}\left(d_{n-1}\right)\right)
$$

for sufficiently large $n$. Finally

$$
A_{n}+B_{n}=\mathcal{O}\left(\frac{1}{n^{\gamma}}\right)
$$

Lemma 3.2 is proved. 
In the next two lemmas we estimate the first and second derivatives of $\widetilde{\Upsilon}_{n}$ and $\widehat{\Upsilon}_{n}$ in the case of $\gamma \in(1,+\infty)$. These estimates will be used in the next section to approximate relative cordinates with Möbius transformations in $C^{2}$-topology for $\gamma>1$. Note that in this case according to Theorem [2.3, $f^{\prime}$ is differentiable on $\left[\xi_{0}, \xi_{0}+1\right]$ (here $f^{\prime}\left(\xi_{0}\right)=f^{\prime}\left(\xi_{0}+0\right)$ and $\left.f^{\prime}\left(\xi_{0}+1\right)=f^{\prime}\left(\xi_{0}-0\right)\right)$ and the modulus of continuity of $f^{\prime \prime}$ is $\mathcal{P}_{\gamma}$.

Lemma 3.3. Let $f \in \mathrm{D}^{1+\mathcal{Z}_{\gamma}}\left(\mathbb{S}^{1} \backslash\left\{\xi_{0}\right\}\right)$ and $\gamma \in(1,+\infty)$. Then there exists a constant $C>0$ such that

$$
\max _{z_{0} \in[0,1]}\left|\frac{\mathrm{d} \widetilde{\Upsilon}_{n}\left(z_{0}\right)}{\mathrm{d} z_{0}}\right| \leq \frac{C}{n^{\gamma-1}}, \quad \max _{z_{0} \in[0,1]}\left|\frac{\mathrm{d} \widehat{\Upsilon}_{n}\left(\bar{z}_{0}\right)}{\mathrm{d} \bar{z}_{0}}\right| \leq \frac{C}{n^{\gamma-1}}
$$

for all $n \geq 1$.

Proof. We prove only the first inequality, the second one can be proved analogously. The same manner as in proof of Lemma 3.2 we can show that

$$
\begin{aligned}
\left|\frac{\mathrm{d} \widetilde{\Upsilon}_{n}\left(z_{0}\right)}{\mathrm{d} z_{0}}\right| & \leq e^{\nu}\left|\sum_{i=0}^{q_{n}-1}\left(\alpha_{i}-\beta_{i}\right) \frac{\mathrm{d} \Psi\left(x_{i}\right)}{\mathrm{d} x_{i}}\right| \leq C e^{\nu} \Omega\left(d_{n-1}, \gamma\right) \sum_{i=0}^{q_{n}-1}\left|\alpha_{i}-\beta_{i}\right|\left|\frac{\mathrm{d} \mathcal{R}_{\alpha_{i}}\left(x_{i}\right)}{\mathrm{d} x_{i}}\right| \\
& +\frac{e^{\nu}}{\inf _{\mathbb{S}^{1}} f^{\prime}(\xi)} \sum_{i=0}^{q_{n}-1}\left|\alpha_{i}-\beta_{i}\right|\left|\frac{\mathrm{d} \mathcal{R}_{\alpha_{i}}\left(x_{i}\right)}{\mathrm{d} x_{i}}-\frac{\mathrm{d} \mathcal{R}_{\beta_{i}}\left(x_{i}\right)}{\mathrm{d} x_{i}}\right|:=C_{n}+D_{n} .
\end{aligned}
$$

Below we estimate $C_{n}$ and $D_{n}$. Since $f^{\prime}$ is differentiable, it can be shown easily

$$
\begin{gathered}
\left|\alpha_{i}-\beta_{i}\right|\left|\frac{\mathrm{d} \mathcal{R}_{\alpha_{i}}\left(x_{i}\right)}{\mathrm{d} x_{i}}\right|=\left|\alpha_{i}-\beta_{i}\right|\left|\frac{f^{\prime}\left(x_{i}\right)-\mathcal{R}_{\alpha_{i}}\left(x_{i}\right)}{x_{i}-\alpha_{i}}\right|= \\
\left|\alpha_{i}-\beta_{i}\right|\left|\int_{\alpha_{i}}^{x_{i}} \frac{f^{\prime \prime}(y)\left(y-\alpha_{i}\right)}{\left(x_{i}-\alpha_{i}\right)^{2}} d y\right|=\mathcal{O}\left(\left|\alpha_{i}-\beta_{i}\right|\right) .
\end{gathered}
$$

Thereby

$$
C_{n}=\mathcal{O}\left(\Omega\left(d_{n-1}, \gamma\right) \sum_{i=0}^{q_{n}-1}\left|\alpha_{i}-\beta_{i}\right|\right)=\mathcal{O}\left(\Omega\left(d_{n-1}, \gamma\right)\right)
$$

Now we estimate $D_{n}$. According to Lemma 2.9 we have

$$
\left|\frac{\mathrm{d} \mathcal{R}_{\alpha_{i}}\left(x_{i}\right)}{\mathrm{d} x_{i}}-\frac{\mathrm{d} \mathcal{R}_{\beta_{i}}\left(x_{i}\right)}{\mathrm{d} x_{i}}\right| \leq C \mathcal{P}_{\gamma}\left(\left|I_{i}^{n-1}\right|\right) .
$$

Hence

$$
D_{n}=\mathcal{O}\left(\sum_{i=0}^{q_{n}-1}\left|\alpha_{i}-\beta_{i}\right| \mathcal{P}_{\gamma}\left(\left|I_{i}^{n-1}\right|\right)\right)=\mathcal{O}\left(\mathcal{P}_{\gamma}\left(d_{n-1}\right)\right) .
$$

From the definiteness of $\mathcal{P}_{\gamma}$

$$
\mathcal{P}_{\gamma}\left(d_{n-1}\right)=\sum_{k=1}^{\infty} \mathcal{Z}_{\gamma}\left(2^{-k} d_{n-1}\right) \leq \int_{0}^{1} \frac{\mathcal{Z}_{\gamma}\left(y d_{n-1}\right)}{y} d y=\frac{1}{\gamma-1}\left(\log \frac{1}{d_{n-1}}\right)^{1-\gamma} .
$$

As before, using the relation $d_{n}=\mathcal{O}\left(\lambda^{n}\right)$ we obtain

$$
\left(\log \frac{1}{d_{n-1}}\right)^{1-\gamma}+\Omega\left(d_{n-1}, \gamma\right)=\mathcal{O}\left(\frac{1}{n^{\gamma-1}}\right) .
$$


Finally

$$
C_{n}+D_{n}=\mathcal{O}\left(\frac{1}{n^{\gamma-1}}\right)
$$

Lemma 3.3 is proved.

Lemma 3.4. Let $f \in \mathrm{D}^{1+\mathcal{Z}_{\gamma}}\left(\mathbb{S}^{1} \backslash\left\{\xi_{0}\right\}\right)$ and $\gamma \in(1,+\infty)$. Then there exists a constant $C>0$ and a natural number $N_{0}=N_{0}(f)$ such that

$$
\max _{z_{0} \in[0,1]}\left|z_{0}\left(1-z_{0}\right) \frac{\mathrm{d}^{2} \widetilde{\Upsilon}_{n}\left(z_{0}\right)}{\mathrm{d} z_{0}^{2}}\right| \leq \frac{C}{n^{\gamma-1}}, \quad \max _{\bar{z}_{0} \in[0,1]}\left|\bar{z}_{0}\left(1-\bar{z}_{0}\right) \frac{\mathrm{d}^{2} \widehat{\Upsilon}_{n}\left(\bar{z}_{0}\right)}{\mathrm{d} \bar{z}_{0}^{2}}\right| \leq \frac{C}{n^{\gamma-1}}
$$

for all $n \geq N_{0}$.

Proof. As above we prove only the first inequality, the second one can be proved analogously. By differentiating (23) and (24) we obtain

$$
\frac{\mathrm{d}^{2} \widetilde{\Upsilon}_{n}\left(z_{0}\right)}{\mathrm{d} z_{0}^{2}}=\left(\alpha_{0}-\beta_{0}\right)^{2} \cdot \frac{\mathrm{d}^{2} \widetilde{\Upsilon}_{n}(x)}{\mathrm{d} x^{2}}
$$

and

$$
\frac{\mathrm{d}^{2} \Psi\left(x_{i}\right)}{\mathrm{d} x^{2}}=\frac{\mathrm{d}^{2} \Psi\left(x_{i}\right)}{\mathrm{d} x_{i}^{2}} \cdot\left(\frac{\mathrm{d} x_{i}}{\mathrm{~d} x}\right)^{2}+\frac{\mathrm{d} \Psi\left(x_{i}\right)}{\mathrm{d} x_{i}} \cdot \frac{\mathrm{d}^{2} x_{i}}{\mathrm{~d} x^{2}} .
$$

Thus

$$
\begin{gathered}
z_{0}\left(1-z_{0}\right) \frac{\mathrm{d}^{2} \widetilde{\Upsilon}_{n}\left(z_{0}\right)}{\mathrm{d} z_{0}^{2}}=z_{0}\left(1-z_{0}\right)\left(\alpha_{0}-\beta_{0}\right)^{2} \sum_{i=0}^{q_{n}-1} \frac{\mathrm{d}^{2} \Psi\left(x_{i}\right)}{\mathrm{d} x_{i}^{2}} \cdot\left(\frac{\mathrm{d} x_{i}}{\mathrm{~d} x}\right)^{2}+ \\
z_{0}\left(1-z_{0}\right)\left(\alpha_{0}-\beta_{0}\right)^{2} \sum_{i=0}^{q_{n}-1} \frac{\mathrm{d} \Psi\left(x_{i}\right)}{\mathrm{d} x_{i}} \cdot \frac{\mathrm{d}^{2} x_{i}}{\mathrm{~d} x^{2}}:=E_{n}+F_{n} .
\end{gathered}
$$

Next we estimate $E_{n}$ and $F_{n}$. Using (25) we get

$$
z_{0}\left(1-z_{0}\right)\left(\alpha_{0}-\beta_{0}\right)^{2}\left(\frac{\mathrm{d} x_{i}}{\mathrm{~d} x}\right)^{2} \leq e^{4 \nu} z_{i}\left(1-z_{i}\right)\left(\alpha_{i}-\beta_{i}\right)^{2}
$$

for any $0 \leq i<q_{n}$. By differentiating (27) we obtain

$$
\frac{\mathrm{d}^{2} \Psi\left(x_{i}\right)}{\mathrm{d} x_{i}^{2}}=-\frac{1}{\mathcal{R}_{\alpha_{i}}^{2}\left(x_{i}\right)} \cdot\left(\frac{\mathrm{d} \mathcal{R}_{\alpha_{i}}\left(x_{i}\right)}{\mathrm{d} x_{i}}\right)^{2}+\frac{1}{\mathcal{R}_{\alpha_{i}}\left(x_{i}\right)} \cdot \frac{\mathrm{d}^{2} \mathcal{R}_{\alpha_{i}}\left(x_{i}\right)}{\mathrm{d} x_{i}^{2}}+
$$

$$
\begin{gathered}
\frac{1}{\mathcal{R}_{\beta_{i}}^{2}\left(x_{i}\right)} \cdot\left(\frac{\mathrm{d} \mathcal{R}_{\beta_{i}}\left(x_{i}\right)}{\mathrm{d} x_{i}}\right)^{2}-\frac{1}{\mathcal{R}_{\beta_{i}}\left(x_{i}\right)} \cdot \frac{\mathrm{d}^{2} \mathcal{R}_{\beta_{i}}\left(x_{i}\right)}{\mathrm{d} x_{i}^{2}}= \\
\left(\frac{1}{\mathcal{R}_{\beta_{i}}^{2}\left(x_{i}\right)}-\frac{1}{\mathcal{R}_{\alpha_{i}}^{2}\left(x_{i}\right)}\right) \cdot\left(\frac{\mathrm{d} \mathcal{R}_{\alpha_{i}}\left(x_{i}\right)}{\mathrm{d} x_{i}}\right)^{2}+\left(\left(\frac{\mathrm{d} \mathcal{R}_{\beta_{i}}\left(x_{i}\right)}{\mathrm{d} x_{i}}\right)^{2}-\left(\frac{\mathrm{d} \mathcal{R}_{\alpha_{i}}\left(x_{i}\right)}{\mathrm{d} x_{i}}\right)^{2}\right) \cdot \frac{1}{\mathcal{R}_{\beta_{i}}^{2}\left(x_{i}\right)}+ \\
\left(\frac{1}{\mathcal{R}_{\alpha_{i}}\left(x_{i}\right)}-\frac{1}{\mathcal{R}_{\beta_{i}}\left(x_{i}\right)}\right) \cdot \frac{\mathrm{d}^{2} \mathcal{R}_{\alpha_{i}}\left(x_{i}\right)}{\mathrm{d} x_{i}^{2}}+\left(\frac{\mathrm{d}^{2} \mathcal{R}_{\alpha_{i}}\left(x_{i}\right)}{\mathrm{d} x_{i}^{2}}-\frac{\mathrm{d}^{2} \mathcal{R}_{\beta_{i}}\left(x_{i}\right)}{\mathrm{d} x_{i}^{2}}\right) \cdot \frac{1}{\mathcal{R}_{\beta_{i}}\left(x_{i}\right)}:=E_{n}^{(1)}+E_{n}^{(2)}+E_{n}^{(3)}+E_{n}^{(4)} .
\end{gathered}
$$

We multiply each $E_{n}^{(s)}, s=1,2,3,4$ to the right hand side of (32) and estimate them separately. Relations (28) and (31) imply

$$
e^{4 v} \sum_{i=0}^{q_{n}-1} z_{i}\left(1-z_{i}\right)\left(\alpha_{i}-\beta_{i}\right)^{2}\left|E_{n}^{(1)}\right| \leq C d_{n-1} \Omega\left(d_{n-1}, \gamma\right) .
$$


From the second inequality of Lemma 2.9 it follows

$$
e^{4 v} \sum_{i=0}^{q_{n}-1} z_{i}\left(1-z_{i}\right)\left(\alpha_{i}-\beta_{i}\right)^{2}\left|E_{n}^{(2)}\right| \leq C d_{n-1} \mathcal{P}_{\gamma}\left(d_{n-1}\right) .
$$

As we have seen in the proof of Lemma 2.9, we have

$$
\frac{\mathrm{d}^{2} \mathcal{R}_{\alpha_{i}}\left(x_{i}\right)}{\mathrm{d} x_{i}^{2}}=2 \int_{\alpha_{i}}^{x_{i}} \frac{\left(f^{\prime \prime}\left(x_{i}\right)-f^{\prime \prime}(y)\right)\left(y-\alpha_{i}\right)}{\left(x_{i}-\alpha_{i}\right)^{3}} d y
$$

By definition of $\left(1-z_{i}\right)$ and the second inequality of Lemma 2.9, we obtain

$$
\left|\left(1-z_{i}\right)\left(\alpha_{i}-\beta_{i}\right) \frac{\mathrm{d}^{2} \mathcal{R}_{\alpha_{i}}\left(x_{i}\right)}{\mathrm{d} x_{i}^{2}}\right| \leq 2 \int_{\alpha_{i}}^{x_{i}} \frac{\left|f^{\prime \prime}\left(x_{i}\right)-f^{\prime \prime}(y)\right|\left(y-\alpha_{i}\right)}{\left(x_{i}-\alpha_{i}\right)^{2}} d y \leq C \mathcal{P}_{\gamma}\left(\left|I_{i}^{n-1}\right|\right) .
$$

Therefore, the last two relations and inequality (28) imply

$$
e^{4 v} \sum_{i=0}^{q_{n}-1} z_{i}\left(1-z_{i}\right)\left(\alpha_{i}-\beta_{i}\right)^{2}\left|E_{n}^{(3)}\right| \leq C \mathcal{P}_{\gamma}\left(d_{n-1}\right) \Omega\left(d_{n-1}, \gamma\right)
$$

From the first inequality of Lemma 2.9 it follows

$$
\begin{gathered}
e^{4 v} \sum_{i=0}^{q_{n}-1} z_{i}\left(1-z_{i}\right)\left(\alpha_{i}-\beta_{i}\right)^{2}\left|E_{n}^{(4)}\right| \leq \\
C \sum_{i=0}^{q_{n}-1}\left(x_{i}-\alpha_{i}\right)\left(\beta_{i}-x_{i}\right)\left|\frac{\mathrm{d}^{2} \mathcal{R}_{\alpha_{i}}\left(x_{i}\right)}{\mathrm{d} x_{i}^{2}}-\frac{\mathrm{d}^{2} \mathcal{R}_{\beta_{i}}\left(x_{i}\right)}{\mathrm{d} x_{i}^{2}}\right| \leq C \mathcal{P}_{\gamma}\left(d_{n-1}\right) .
\end{gathered}
$$

Relations (32)-(37) then imply the following estimate

$$
\left|E_{n}\right|=\mathcal{O}\left(\frac{1}{n^{\gamma-1}}\right)
$$

We pass to estimate $F_{n}$. First we show the validity of the following inequalities

$$
e^{-2 \nu} \inf _{x \in \mathbb{S}^{1}}\left|\frac{f^{\prime \prime}(x)}{f^{\prime}(x)}\right| \cdot\left|\alpha_{i}-\beta_{i}\right| \leq\left(\alpha_{0}-\beta_{0}\right)^{2}\left|\frac{\mathrm{d}^{2} x_{i}}{\mathrm{~d} x^{2}}\right| \leq e^{2 \nu} \sup _{x \in \mathbb{S}^{1}}\left|\frac{f^{\prime \prime}(x)}{f^{\prime}(x)}\right| \cdot\left|\alpha_{i}-\beta_{i}\right|
$$

for any $0 \leq i<q_{n}$. Consider the function

$$
\mathcal{H}_{i}(x)=\sum_{j=0}^{i-1}\left(f^{j}(x)\right)^{\prime}
$$

where $x \in I_{0}^{n-1}$ and $0 \leq i<q_{n}$. Using Finzi's inequality it can be easily shown

$$
e^{-\nu} \leq \frac{\mathcal{H}_{i}(x)}{\mathcal{H}_{i}(y)} \leq e^{\nu}
$$

for any $x, y \in I_{0}^{n-1}$ and $0 \leq i<q_{n}$. On the other hand

$$
\int_{I_{0}^{n-1}} \mathcal{H}_{i}(x) d x=\sum_{j=0}^{i-1}\left|I_{j}^{n-1}\right| .
$$


This and inequality (39) imply

$$
e^{-\nu} \frac{1}{\left|I_{0}^{n-1}\right|} \sum_{j=0}^{i-1}\left|I_{j}^{n-1}\right| \leq \mathcal{H}_{i}(x) \leq e^{\nu} \frac{1}{\left|I_{0}^{n-1}\right|} \sum_{j=0}^{i-1}\left|I_{j}^{n-1}\right| .
$$

We find $\frac{\mathrm{d}^{2} x_{i}}{\mathrm{~d} x^{2}}$ :

$$
\frac{\mathrm{d}^{2} x_{i}}{\mathrm{~d} x^{2}}=\left(f^{i}(x)\right)^{\prime} \sum_{j=0}^{i-1} \frac{f^{\prime \prime}\left(x_{j}\right)}{f^{\prime}\left(x_{j}\right)}\left(f^{j}(x)\right)^{\prime} .
$$

Inequalities (25) and (40) imply

$$
\left(\alpha_{0}-\beta_{0}\right)^{2}\left|\frac{\mathrm{d}^{2} x_{i}}{\mathrm{~d} x^{2}}\right| \leq \sup _{x \in \mathbb{S}^{1}}\left|\frac{f^{\prime \prime}(x)}{f^{\prime}(x)}\right|\left(\alpha_{0}-\beta_{0}\right)^{2}\left(f^{i}(x)\right)^{\prime} \mathcal{H}_{i}(x) \leq e^{2 \nu} \sup _{x \in \mathbb{S}^{1}}\left|\frac{f^{\prime \prime}(x)}{f^{\prime}(x)}\right| \cdot\left|\alpha_{i}-\beta_{i}\right| .
$$

The left hand side of (38) is proved similarly. Now we continue estimating $F_{n}$. After above preparations we have

$$
\left|F_{n}\right| \leq C \sum_{i=0}^{q_{n}-1} z_{i}\left(1-z_{i}\right)\left|\alpha_{i}-\beta_{i}\right|\left|\frac{\mathrm{d} \Psi\left(x_{i}\right)}{\mathrm{d} x_{i}}\right| .
$$

This and inequalities (27)-(30) imply

$$
\left|F_{n}\right| \leq C\left(A_{n}+B_{n}\right) .
$$

As we have shown in the proof of Lemma 3.2

$$
A_{n}+B_{n}=\mathcal{O}\left(\frac{1}{n^{\gamma}}\right)
$$

for sufficiently large $n$. Therefore

$$
\left|F_{n}\right| \leq \frac{C}{n^{\gamma}}
$$

for sufficiently large $n$. Finally we have

$$
\left|E_{n}\right|+\left|F_{n}\right|=\mathcal{O}\left(\frac{1}{n^{\gamma-1}}\right)
$$

for sufficiently large $n$. Lemma 3.4 is therefore completely proved.

\section{Comparing relative coordinates with Möbius transforma- tions}

In this section we show that the relative coordinates $z_{q_{n}}\left(z_{0}\right)$ and $\hat{z}_{q_{n-1}}\left(\bar{z}_{0}\right)$ are approximated by Möbius transformations. To characterize these approximations we define a Möbius map $\mathcal{M}_{\mathcal{T}}$ as follows

$$
\mathcal{M}_{\mathcal{T}}(z)=\frac{z \mathcal{T}}{1+z(\mathcal{T}-1)}
$$


Lemma 4.1. Let $f \in \mathrm{D}^{1+\mathcal{Z}_{\gamma}}\left(\mathbb{S}^{1} \backslash\left\{\xi_{0}\right\}\right)$ and $\gamma \in(0,1]$. Then there exists a constant $C>0$ and a natural number $N_{0}=N_{0}(f)$ such that

$$
\left\|z_{q_{n}}-\mathcal{M}_{\widetilde{m}_{n}}\right\|_{C^{1}([0,1])} \leq \frac{C}{n^{\gamma}}, \quad\left\|\hat{z}_{q_{n-1}}-\mathcal{M}_{\widehat{m}_{n}}\right\|_{C^{1}([0,1])} \leq \frac{C}{n^{\gamma}}
$$

for all $n \geq N_{0}$.

Proof. We prove the first inequality. For this we find the explicit form of $z_{q_{n}}\left(z_{0}\right)$. After simple computations we get

$$
\frac{1-z_{q_{n}}}{z_{q_{n}}} \cdot \frac{z_{0}}{1-z_{0}}=\frac{\mathcal{R}\left(\left[\xi_{q_{n-1}}, x\right] ; f^{q_{n}}\right)}{\mathcal{R}\left(\left[x, \xi_{0}\right] ; f^{q_{n}}\right)} .
$$

On the other hand, the relation (13) implies

$$
\frac{\mathcal{R}\left(\left[\xi_{q_{n-1}}, x\right] ; f^{q_{n}}\right)}{\mathcal{R}\left(\left[x, \xi_{0}\right] ; f^{q_{n}}\right)}=\frac{1}{\widetilde{m}_{n}} \exp \left(\widetilde{\Upsilon}_{n}\left(z_{0}\right)\right)
$$

Therefore, the last two relations imply

$$
\frac{1-z_{q_{n}}}{z_{q_{n}}} \cdot \frac{z_{0}}{1-z_{0}}=\frac{1}{\widetilde{m}_{n}} \exp \left(\widetilde{\Upsilon}_{n}\left(z_{0}\right)\right) .
$$

Solving for $z_{q_{n}}$, we get

$$
z_{q_{n}}\left(z_{0}\right)=\frac{z_{0} \widetilde{m}_{n}}{\left(1-z_{0}\right) \exp \left(\widetilde{\Upsilon}_{n}\left(z_{0}\right)\right)+z_{0} \widetilde{m}_{n}} .
$$

Using Lemma 3.1 we get

$$
\max _{z_{0} \in[0,1]}\left|z_{q_{n}}\left(z_{0}\right)-\mathcal{M}_{\widetilde{m}_{n}}\left(z_{0}\right)\right| \leq \frac{C}{n^{\gamma}}
$$

for all $n \geq 1$. By differentiating (42) we obtain

$$
z_{q_{n}}^{\prime}\left(z_{0}\right)=\frac{\left(1-z_{0}\left(1-z_{0}\right) \widetilde{\Upsilon}_{n}^{\prime}\left(z_{0}\right)\right) \widetilde{m}_{n} \exp \left(\widetilde{\Upsilon}_{n}\left(z_{0}\right)\right)}{\left(\left(1-z_{0}\right) \exp \left(\widetilde{\Upsilon}_{n}\left(z_{0}\right)\right)+z_{0} \widetilde{m}_{n}\right)^{2}}
$$

Utilizing Lemma 3.2

$$
\max _{z_{0} \in[0,1]}\left|z_{q_{n}}^{\prime}\left(z_{0}\right)-\mathcal{M}_{\widetilde{m}_{n}}^{\prime}\left(z_{0}\right)\right| \leq \frac{C}{n^{\gamma}}
$$

for all $n \geq N_{0}$. Inequalities (43) and (44) imply the proof of first inequality of Lemma 4.1. The proof of the second inequality is similar.

Now we consider the case $\gamma>1$.

Lemma 4.2. Let $f \in \mathrm{D}^{1+\mathcal{Z}_{\gamma}}\left(\mathbb{S}^{1} \backslash\left\{\xi_{0}\right\}\right)$ and $\gamma>1$. Then there exists a constant $C>0$ and natural number $N_{0}=N_{0}(f)$ such that for all $n \geq N_{0}$ the following inequalities hold

$$
\left\|z_{q_{n}}-\mathcal{M}_{m_{n}}\right\|_{C^{1}([0,1])} \leq \frac{C}{n^{\gamma}}, \quad\left\|\hat{z}_{q_{n-1}}-\mathcal{M}_{\frac{c_{n}}{m_{n}}}\right\|_{C^{1}([0,1])} \leq \frac{C}{n^{\gamma}} .
$$

Moreover,

$$
\left\|z_{q_{n}}^{\prime \prime}-\mathcal{M}_{m_{n}}^{\prime \prime}\right\|_{C^{0}([0,1])} \leq \frac{C}{n^{\gamma-1}}, \quad\left\|\hat{z}_{q_{n-1}}^{\prime \prime}-\mathcal{M}_{\frac{c_{n}}{m_{n}}}^{\prime \prime}\right\|_{C^{0}([0,1])} \leq \frac{C}{n^{\gamma-1}}
$$


Proof. To prove this lemma we use the explicit forms of $z_{q_{n}}\left(z_{0}\right), z_{q_{n}}^{\prime}\left(z_{0}\right)$ and $\hat{z}_{q_{n-1}}\left(\bar{z}_{0}\right)$, $\hat{z}_{q_{n-1}}^{\prime}\left(\bar{z}_{0}\right)$. As we have shown above

$$
\begin{gathered}
z_{q_{n}}\left(z_{0}\right)=\frac{z_{0} \widetilde{m}_{n}}{\left(1-z_{0}\right) \exp \left(\widetilde{\Upsilon}_{n}\left(z_{0}\right)\right)+z_{0} \widetilde{m}_{n}}, \\
z_{q_{n}}^{\prime}\left(z_{0}\right)=\frac{\left(1-z_{0}\left(1-z_{0}\right) \widetilde{\Upsilon}_{n}^{\prime}\left(z_{0}\right)\right) \widetilde{m}_{n} \exp \left(\widetilde{\Upsilon}_{n}\left(z_{0}\right)\right)}{\left(\left(1-z_{0}\right) \exp \left(\widetilde{\Upsilon}_{n}\left(z_{0}\right)\right)+z_{0} \widetilde{m}_{n}\right)^{2}} .
\end{gathered}
$$

The same manner as in the proof of above lemma, it can be found

$$
\hat{z}_{q_{n-1}}\left(\bar{z}_{0}\right)=\frac{\bar{z}_{0} \widehat{m}_{n}}{\left(1-\bar{z}_{0}\right) \exp \left(\widehat{\Upsilon}_{n}\left(\bar{z}_{0}\right)\right)+\bar{z}_{0} \widehat{m}_{n}}
$$

By differentiating this we obtain

$$
\hat{z}_{q_{n-1}}^{\prime}\left(\bar{z}_{0}\right)=\frac{\left(1-\bar{z}_{0}\left(1-\bar{z}_{0}\right) \widehat{\Upsilon}_{n}^{\prime}\left(\bar{z}_{0}\right)\right) \widehat{m}_{n} \exp \left(\widehat{\Upsilon}_{n}\left(\bar{z}_{0}\right)\right)}{\left(\left(1-\bar{z}_{0}\right) \exp \left(\widehat{\Upsilon}_{n}\left(\bar{z}_{0}\right)\right)+\bar{z}_{0} \widehat{m}_{n}\right)^{2}} .
$$

Now we compare $\widetilde{m}_{n}, \widehat{m}_{n}$ with $m_{n}, c_{n} m_{n}^{-1}$ respectively. By assumption of lemma $\gamma>1$, therefore according to Theorem 2.3, $f^{\prime}$ is differentiable. Thus

$$
\left|\log \widetilde{m}_{n}-\log m_{n}\right| \leq \frac{1}{2} \sum_{i=0}^{q_{n}-1} \int_{I_{i}^{n-1}}\left|\frac{f^{\prime \prime}(x)}{f^{\prime}\left(\xi_{i}\right)}-\frac{f^{\prime \prime}(x)}{f^{\prime}(x)}\right| d x .
$$

Theorem 2.1 implies

$$
\int_{I_{i}^{n-1}}\left|\frac{f^{\prime \prime}(x)}{f^{\prime}\left(\xi_{i}\right)}-\frac{f^{\prime \prime}(x)}{f^{\prime}(x)}\right| d x \leq \frac{\Omega\left(d_{n-1}, \gamma\right)}{\left(\inf _{\mathbb{S}^{1}} f^{\prime}(x)\right)^{2}} \int_{I_{i}^{n-1}}\left|f^{\prime \prime}(x)\right| d x .
$$

It is clear that $\Omega\left(d_{n-1}, \gamma\right)=\mathcal{O}\left(\lambda^{n}\right)$ for $\gamma>1$. Hence

$$
\widetilde{m}_{n}=m_{n}+\mathcal{O}\left(\lambda^{n}\right) .
$$

Similarly it can be shown

$$
\widehat{m}_{n}=\exp \left((-1)^{n} \sum_{j=0}^{q_{n-1}-1} \int_{I_{j}^{n}} \frac{f^{\prime \prime}(x)}{2 f^{\prime}(x)} d x\right)+\mathcal{O}\left(\lambda^{n}\right) .
$$

On the other hand

$$
m_{n} \cdot \exp \left((-1)^{n} \sum_{j=0}^{q_{n-1}-1} \int_{I_{j}^{n}} \frac{f^{\prime \prime}(x)}{2 f^{\prime}(x)} d x\right)=\exp \left((-1)^{n} \int_{\mathbb{S}^{1}} \frac{f^{\prime \prime}(x)}{2 f^{\prime}(x)} d x\right)=c_{n} .
$$

Therefore

$$
\widetilde{m}_{n}=\frac{c_{n}}{m_{n}}+\mathcal{O}\left(\lambda^{n}\right)
$$


Finally, relations (47)-(52) together with Lemmas 3.1 and 3.2 imply the first assertion of Lemma 4.2. To prove the second assertion, we find the explicit forms of $z_{q_{n}}^{\prime \prime}$ and $\hat{z}_{q_{n-1}}^{\prime \prime}$ as follows

$$
\begin{aligned}
& z_{q_{n}}^{\prime \prime}\left(z_{0}\right)=\frac{\widetilde{m}_{n} \exp \left(\widetilde{\Upsilon}_{n}\left(z_{0}\right)\right)\left(\widetilde{\Upsilon}_{n}^{\prime}\left(z_{0}\right)\left(2 z_{0}-z_{0}\left(1-z_{0}\right) \widetilde{\Upsilon}_{n}^{\prime}\left(z_{0}\right)\right)-z_{0}\left(1-z_{0}\right) \widetilde{\Upsilon}_{n}^{\prime \prime}\left(z_{0}\right)\right)}{\left(\left(1-z_{0}\right) \exp \left(\widetilde{\Upsilon}_{n}\left(z_{0}\right)\right)+z_{0} \widetilde{m}_{n}\right)^{2}}- \\
& \frac{2 \widetilde{m}_{n} \exp \left(\widetilde{\Upsilon}_{n}\left(z_{0}\right)\right)\left(1-z_{0}\left(1-z_{0}\right) \widetilde{\Upsilon}_{n}^{\prime}\left(z_{0}\right)\right)\left(\widetilde{m}_{n}-\exp \left(\widetilde{\Upsilon}_{n}\left(z_{0}\right)\right)+\left(1-z_{0}\right) \widetilde{\Upsilon}_{n}^{\prime}\left(z_{0}\right)\right)}{\left(\left(1-z_{0}\right) \exp \left(\widetilde{\Upsilon}_{n}\left(z_{0}\right)\right)+z_{0} \widetilde{m}_{n}\right)^{3}} .
\end{aligned}
$$

Similarly

$$
\begin{aligned}
& \hat{z}_{q_{n}}^{\prime \prime}\left(\bar{z}_{0}\right)=\frac{\widehat{m}_{n} \exp \left(\widehat{\Upsilon}_{n}\left(\bar{z}_{0}\right)\right)\left(\widehat{\Upsilon}_{n}^{\prime}\left(\bar{z}_{0}\right)\left(2 \bar{z}_{0}-\bar{z}_{0}\left(1-\bar{z}_{0}\right) \widehat{\Upsilon}_{n}^{\prime}\left(\bar{z}_{0}\right)\right)-\bar{z}_{0}\left(1-\bar{z}_{0}\right) \widehat{\Upsilon}_{n}^{\prime \prime}\left(\bar{z}_{0}\right)\right)}{\left(\left(1-\bar{z}_{0}\right) \exp \left(\widehat{\Upsilon}_{n}\left(\bar{z}_{0}\right)\right)+\bar{z}_{0} \widehat{m}_{n}\right)^{2}}- \\
& \frac{2 \widehat{m}_{n} \exp \left(\widehat{\Upsilon}_{n}\left(\bar{z}_{0}\right)\right)\left(1-\bar{z}_{0}\left(1-\bar{z}_{0}\right) \widehat{\Upsilon}_{n}^{\prime}\left(\bar{z}_{0}\right)\right)\left(\widehat{m}_{n}-\exp \left(\widehat{\Upsilon}_{n}\left(\bar{z}_{0}\right)\right)+\left(1-\bar{z}_{0}\right) \widehat{\Upsilon}_{n}^{\prime}\left(\bar{z}_{0}\right)\right)}{\left(\left(1-\bar{z}_{0}\right) \exp \left(\widehat{\Upsilon}_{n}\left(\bar{z}_{0}\right)\right)+\bar{z}_{0} \widehat{m}_{n}\right)^{3}} .
\end{aligned}
$$

Using relations (51), (52) together with Lemmas 3.1, 3.2, 3.3 and 3.4 we obtain inequalities (46). Lemma 4.2 is therefore completely proved.

\section{Proofs of main theorems}

In this section we provide the proofs of our main theorems. Note that the proofs follow closely that of [18]. The flows of proofs are as follow: first we introduce a new renormalized coordinate $z$ on the $\mathcal{V}_{n}=I_{0}^{n} \cup I_{0}^{n-1}$ and then express the functions $f_{n}$ and $g_{n}$ in terms of renormalized coordinate. Finally, using relations between new renormalized coordinate $z$ and relative coordinates $z_{0}, \bar{z}_{0}$ and applying Lemmas 4.1 , 4.2 we obtain the proof of Theorem 1.2. To prove Theorem 1.3 we utilize Theorems 2.1 and 2.3 .

Proof of Theorem 1.2. Renormalized coordinate $z$ on $\mathcal{V}_{n}$ is defined by

$$
x=\xi_{0}+z\left(\xi_{0}-\xi_{q_{n-1}}\right) .
$$

In this new coordinate the points $\xi_{q_{n-1}}$ and $\xi_{0}$ go to $(-1)$ and 0 respectively. Denote by $a_{n}$ and $\left(-b_{n}\right)$ the new coordinates of points $\xi_{q_{n}}$ and $\xi_{q_{n}+q_{n-1}}$ i.e.,

$$
a_{n}=\frac{\xi_{q_{n}}-\xi_{0}}{\xi_{0}-\xi_{q_{n-1}}}, \quad-b_{n}=\frac{\xi_{q_{n}+q_{n-1}}-\xi_{0}}{\xi_{0}-\xi_{q_{n-1}}}
$$

It is clear that for any $x \in\left[\xi_{q_{n-1}}, \xi_{0}\right]$ there exists a unique relative coordinate $z_{0} \in[0,1]$ and renormalized coordinate $z \in[-1,0]$ which correspond to $x$. Similarly, for any $y \in$ $\left[\xi_{0}, \xi_{q_{n}}\right]$ there exists $\bar{z}_{0} \in[0,1]$ and $z \in\left[0, a_{n}\right]$. Using definitions of relative coordinate and renormalized coordinate one can show that

$$
z_{0}=-z, \quad \bar{z}_{0}=1-\frac{z}{a_{n}} .
$$


By definiteness of $f_{n}$ and the explicit form of $z_{q_{n}}$, we have

$$
\begin{gathered}
f_{n}(z)=\frac{f^{q_{n}}(x)-\xi_{0}}{\xi_{0}-\xi_{q_{n-1}}}=\frac{\xi_{q_{n}}-\xi_{0}}{\xi_{0}-\xi_{q_{n-1}}}-\frac{\xi_{q_{n}}-f^{q_{n}}(x)}{\xi_{q_{n}}-\xi_{q_{n}+q_{n-1}}} \cdot \frac{\xi_{q_{n}}-\xi_{q_{n}+q_{n-1}}}{\xi_{0}-\xi_{q_{n-1}}}= \\
a_{n}-\left(a_{n}+b_{n}\right) z_{q_{n}}\left(z_{0}\right)=a_{n}-\left(a_{n}+b_{n}\right) z_{q_{n}}(-z) .
\end{gathered}
$$

Analogously it can be shown, that

$$
g_{n}(z)=-b_{n}-\left(1-b_{n}\right) \hat{z}_{q_{n-1}}\left(1-\frac{z}{a_{n}}\right) .
$$

On the other hand an easy computation shows that the functions $\widetilde{F}_{n}, \widetilde{C}_{n}$ can be represented by Möbius transformations $\mathcal{M}_{\widetilde{m}_{n}}, \mathcal{M}_{\widehat{m}_{n}}$ respectively as follows

$$
\widetilde{F}_{n}(z)=a_{n}-\left(a_{n}+b_{n}\right) \mathcal{M}_{\widetilde{m}_{n}}(-z), \quad \widetilde{C}_{n}(z)=-b_{n}-\left(1-b_{n}\right) \mathcal{M}_{\widehat{m}_{n}}\left(1-\frac{z}{a_{n}}\right) .
$$

Relations (53)-(55) imply

$$
\begin{gathered}
f_{n}(z)-\widetilde{F}_{n}(z)=-\left(a_{n}+b_{n}\right)\left(z_{q_{n}}(-z)-\mathcal{M}_{\widetilde{m}_{n}}(-z)\right), \quad z \in[-1,0], \\
g_{n}(z)-\widetilde{G}_{n}(z)=-\left(1-b_{n}\right)\left(\hat{z}_{q_{n-1}}\left(1-\frac{z}{a_{n}}\right)-\mathcal{M}_{\widehat{m}_{n}}\left(1-\frac{z}{a_{n}}\right)\right), \quad z \in\left[0, a_{n}\right] .
\end{gathered}
$$

By differentiating these we obtain

$$
\begin{gathered}
f_{n}^{\prime}(z)-\widetilde{F}_{n}^{\prime}(z)=\left(a_{n}+b_{n}\right)\left(z_{q_{n}}^{\prime}(-z)-\mathcal{M}_{\widetilde{m}_{n}}^{\prime}(-z)\right), \quad z \in[-1,0], \\
g_{n}^{\prime}(z)-\widetilde{G}_{n}^{\prime}(z)=\frac{1-b_{n}}{a_{n}}\left(\hat{z}_{q_{n-1}}^{\prime}\left(1-\frac{z}{a_{n}}\right)-\mathcal{M}_{\widehat{m}_{n}}^{\prime}\left(1-\frac{z}{a_{n}}\right)\right), \quad z \in\left[0, a_{n}\right] .
\end{gathered}
$$

Using Denjoy's inequality (see [1]) and properties of dynamical partition one can obtain

$$
a_{n}+b_{n} \leq e^{\nu}, \quad \frac{1-b_{n}}{a_{n}} \leq e^{\nu} \quad \text { and } \quad 0<1-b_{n}<1
$$

The last relations together with (56)-(59) and Lemma 4.1 imply

$$
\left\|f_{n}-\widetilde{F}_{n}\right\|_{C^{1}([-1,0])} \leq \frac{C}{n^{\gamma}}, \quad\left\|g_{n}-\widehat{G}_{n}\right\|_{C^{1}\left(\left[0, a_{n}\right]\right)} \leq \frac{C}{n^{\gamma}}
$$

for all $n \geq N_{0}$. Theorem 1.2 is proved.

Proof of Theorem 1.3. From Lemma 3.1 and the relations (51), (52), (56)-(60) directly follow the inequalities (5) of Theorem 1.3. To prove the inequalities (6) we use Theorem 2.3. According to that theorem, $f^{\prime}$ is differentiable, hence $z_{q_{n}}^{\prime}$ and $\hat{z}_{q_{n-1}}^{\prime}$ are differentiable. By differentiating (58), (59) we obtain

$$
\begin{gathered}
f_{n}^{\prime \prime}(z)-\widetilde{F}_{n}^{\prime \prime}(z)=-\left(a_{n}+b_{n}\right)\left(z_{q_{n}}^{\prime \prime}(-z)-\mathcal{M}_{\widetilde{m}_{n}}^{\prime \prime}(-z)\right), \quad z \in[-1,0] \\
g_{n}^{\prime \prime}(z)-\widetilde{G}_{n}^{\prime \prime}(z)=-\frac{1-b_{n}}{a_{n}^{2}}\left(\hat{z}_{q_{n-1}}^{\prime \prime}\left(1-\frac{z}{a_{n}}\right)-\mathcal{M}_{\widehat{m}_{n}}^{\prime \prime}\left(1-\frac{z}{a_{n}}\right)\right), \quad z \in\left[0, a_{n}\right] .
\end{gathered}
$$


These together with relations (51), (52), (60) and the second assertion of Lemma 4.2 imply

$$
\left\|f_{n}^{\prime \prime}-F_{n}^{\prime \prime}\right\|_{C^{0}([-1,0])} \leq \frac{C}{n^{\gamma-1}}, \quad\left\|g_{n}^{\prime \prime}-G_{n}^{\prime \prime}\right\|_{C^{0}\left(\left[0, a_{n}\right]\right)} \leq \frac{C}{a_{n} n^{\gamma-1}}
$$

for all $n \geq N_{0}$. The inequalities (6) of Theorem 1.3 are proved.

Now we prove the inequality (77). To prove this we use from the relation between $f_{n}$ and $g_{n+1}$. Since these functions correspond to the mapping $f^{q_{n}}$ in different coordinate systems, we have

$$
g_{n+1}(z)=-\frac{1}{a_{n}} f_{n}\left(-a_{n} z\right) .
$$

This relation implies the following two equalities

$$
\begin{gathered}
g_{n+1}^{\prime}(0)=f_{n}^{\prime}(0), \\
g_{n+1}\left(a_{n+1}\right)=-\frac{1}{a_{n}} f_{n}\left(-a_{n} a_{n+1}\right) .
\end{gathered}
$$

Note that $f_{n}^{\prime}(0)$ exists and it is equal to $\left(f^{q_{n}}\left(\xi_{0}-0\right)\right)^{\prime}$ if $n$ is even and $\left(f^{q_{n}}\left(\xi_{0}+0\right)\right)^{\prime}$ if $n$ is odd. Using (47)-(50) and (53), (54) we rewrite (61), (62) as

$$
\begin{gathered}
\frac{\left(1-b_{n+1}\right)}{a_{n+1}} \cdot \frac{\exp \left(\widehat{\Upsilon}_{n+1}(1)\right)}{\widehat{m}_{n+1}}=\frac{\left(a_{n}+b_{n}\right) \widetilde{m}_{n}}{\exp \left(\widetilde{\Upsilon}_{n}(0)\right)}, \\
-b_{n+1}=-1+\frac{\left(a_{n}+b_{n}\right) a_{n+1} \widetilde{m}_{n}}{\left(1-a_{n} a_{n+1}\right) \exp \left(\widetilde{\Upsilon}_{n}\left(a_{n} a_{n+1}\right)\right)+a_{n} a_{n+1} \widetilde{m}_{n}} .
\end{gathered}
$$

From the last equation we find $\left(1-b_{n+1}\right) / a_{n+1}$ and then substituting this expression in (63) we obtain

$$
\frac{\exp \left(\widehat{\Upsilon}_{n+1}(1)+\widetilde{\Upsilon}_{n}(0)\right)}{\widehat{m}_{n+1}}=\left(1-a_{n} a_{n+1}\right) \exp \left(\widetilde{\Upsilon}_{n}\left(a_{n} a_{n+1}\right)\right)+a_{n} a_{n+1} \widetilde{m}_{n}
$$

Using this and (64) we get

$$
\begin{gathered}
\frac{a_{n+1}}{c_{n+1}}+\frac{b_{n+1} \exp \left(\widehat{\Upsilon}_{n+1}(1)+\widetilde{\Upsilon}_{n}(0)\right)}{\widehat{m}_{n+1}}-1= \\
a_{n+1}\left(\frac{1}{c_{n+1}}-a_{n} \exp \left(\widetilde{\Upsilon}_{n}\left(a_{n} a_{n+1}\right)\right)-b_{n} \widetilde{m}_{n}\right)+\exp \left(\widetilde{\Upsilon}_{n}\left(a_{n} a_{n+1}\right)\right)-1 .
\end{gathered}
$$

Utilizing Lemma 3.1 and relations (51) and (52) we can show that

$$
a_{n+1}+b_{n+1} m_{n+1}-c_{n+1}=c_{n+1} a_{n+1}\left(c_{n}-a_{n}-b_{n} m_{n}\right)+\mathcal{O}\left(\frac{1}{n^{\gamma}}\right)
$$

for all $n \geq N_{0}$. Set $r_{n+1}:=a_{n+1}+b_{n+1} m_{n+1}-c_{n+1}$ and $a_{n+2} c_{n+2}:=1$. Iterating relation (65) we get

$$
r_{n+1}=r_{1} \prod_{i=2}^{n+1}\left(-a_{i} c_{i}\right)+\mathcal{O}\left(\sum_{j=3}^{n+2} \prod_{i=j}^{n+2} \frac{\left(-a_{i} c_{i}\right)}{(i-2)^{\gamma}}\right)
$$


It is clear

$$
\left|\prod_{i=j}^{n+1}\left(-a_{i} c_{i}\right)\right| \leq \max \left\{c, \frac{1}{c}\right\} a_{n+1} \cdot \frac{\left|I_{0}^{n}\right|}{\left|I_{0}^{j-1}\right|} .
$$

From well known fact $\frac{\left|I_{0}^{n}\right|}{\left|I_{0}^{j-1}\right|}=\mathcal{O}\left(\lambda^{n-j+1}\right)$ (see, for instance [1]) we obtain

$$
\left|r_{n+1}\right|=\mathcal{O}\left(a_{n+1} \sum_{i=1}^{n} \frac{\lambda^{n-i}}{i^{\gamma}}\right)
$$

On the other hand

$$
\sum_{i=1}^{n} \frac{\lambda^{n-i}}{i^{\gamma}}=\sum_{i=1}^{\left[\frac{n}{2}\right]-1} \frac{\lambda^{n-i}}{i^{\gamma}}+\sum_{i=\left[\frac{n}{2}\right]}^{n} \frac{\lambda^{n-i}}{i^{\gamma}}=\mathcal{O}\left(\left[\frac{n}{2}\right] \lambda^{\left[\frac{n}{2}\right]}+\frac{1}{\left[\frac{n}{2}\right]^{\gamma}}\right)=\mathcal{O}\left(\frac{1}{n^{\gamma}}\right)
$$

where $[\cdot]$ is an integer part of a given number. Hence

$$
\left|r_{n+1}\right|=\mathcal{O}\left(\frac{a_{n+1}}{n^{\gamma}}\right)
$$

Theorem 1.3 is completely proved.

Afterthought. At the end of this work, we would like to give our opinion on the further development of our result. Since the rate of convergence is given in explicit form, we believe that this result will have applications in regularity problem of conjugacy. Of course, for $\gamma \in(0,1 / 2]$ it is difficult to expect the regularity of conjugacy. Because, in this case the second derivative of circle diffeomorphisms can be very "bad" (see [32]). However, in the case $\gamma>1 / 2$ the situation gets better that is, in this case due to Theorem 2.2 (stated in Section 2), $f^{\prime}$ is absolute continuous on $\mathbb{S}^{1} \backslash\left\{\xi_{0}\right\}$ and $f^{\prime \prime} \in L_{p}\left(\mathbb{S}^{1}\right)$ for every $p>1$. Such diffeomorphisms are known as a class of Katznelson \& Ornstein (KO class) in the theory of circle maps. Katznelson \& Ornstein proved that diffeomorphisms from KO class are absolute continuously conjugated with rigid rotation for bounded type of irrational rotation numbers [9]. It is natural to expect analogues result to Katznelson \& Ornstein for conjugacy between two circle diffeomorphisms with breaks. In the case $\gamma>1$, in spite of $f$ belongs to $C^{2}\left(\mathbb{S}^{1} \backslash\left\{\xi_{0}\right\}\right)$, the convergence rate is sub-exponential. Therefore it is difficult to expect $C^{1}$-rigidity. Because, for $C^{1}$-rigidity, it is very important the exponentiality of convergence rate. This point is known from previous works mentioned in Section 1 and explained e.g. in [22]. However, it can be expected the convergence renormalizations with sub-exponential rate.

Acknowledgements. The authors would like to thank professors A. A. Dzhalilov and K. M. Khanin for making several useful suggestions. We also wish to express our thanks to the referee for providing us with helpful comments. The paper is supported via the grants DIP-2014-034 and FRGS/1/2014/ST06/UKM/01/1.

\section{References}

[1] H. Akhadkulov, A. Dzhalilov, D. Mayer, On conjugations of circle homeomorphisms with two break points, Ergodic Theory Dynam. Systems. 34,(3), 725-741, (2014). 
[2] V. I. Arnol'd, Small denominators: I. Mappings from the circle onto itself. Izv. Akad. Nauk SSSR, Ser. Mat., 25, 21-86, (1961).

[3] K. Cunha, D. Smania, Renormalization for piecewise smooth homeomorphisms on the circle. Ann. I. H. Poincaré - AN 30, 441-462, (2013).

[4] A. Dzhalilov, M. S. M. Noorani and H. Akhadkulov, Stronger version of Denjoy-type inequality. Bull. Malays. Math. Sci. Soc. 2,37(4), 939-948, (2014).

[5] M. Herman, Sur la conjugaison différentiable des difféomorphismes du cercle à des rotations. Inst. Hautes Etudes Sci. Publ. Math., 49, 5-234, (1979).

[6] J. Hu and D. Sullivan, Topological Conjugacy of Circle Diffeomorphisms. Ergodic Theory Dynam. Systems 17 no 1, 173-186, (1997).

[7] F. John and L. Nirenberg, On functions of bounded mean oscillation. Comm. Pure Appl. Math. , 14, 415-426, (1961).

[8] Y. Katznelson and D. Ornstein, The differentiability of the conjugation of certain diffeomorphisms of the circle. Ergod. Theor. Dyn. Syst., 9, 643-680, (1989).

[9] Y. Katznelson and D. Ornstein, The absolute continuity of the conjugation of certain diffeomorphisms of the circle. Ergod. Theor. Dyn. Syst., 9, 681-690, (1989).

[10] K. Khanin and D. Khmelev, Renormalizations and rigidity theory for circle homeomorphisms with singularities of break type, Commun. Math. Phys., 235, No. 1, 69-124, (2003).

[11] K. Khanin, S. Kocić, Renormalization conjecture and rigidity theory for circle diffeomorphisms with breaks, Geometric and Functional Analysis, Volume 24, Issue 6, 2002-2028, (2014).

[12] K. Khanin, S. Kocić, E. Mazzeo, $C^{1}$-rigidity of circle diffeomorphisms with breaks for almost all rotation numbers. http://www.ma.utexas.edu/mp_arc/c/11/11-102.pdf

[13] K. Khanin and Ya. Sinai, A New Proof of M. Herman's Theorem. Commun. Math. Phys., 112, 89-101, (1987).

[14] K. Khanin and Ya. Sinai, Smoothness of conjugacies of diffeomorphisms of the circle with rotations. Russ. Math. Surv., 44,69-99, (1989), translation of Usp. Mat. Nauk, 44, 57-82, (1989).

[15] K. Khanin, A. Teplinsky, Robust rigidity for diffeomorphisms with singularities. Invent. Math. 169, 193-218, (2007).

[16] K. Khanin, A. Teplinsky, Renormalization Horseshoe and Rigidity for Circle Diffeomorphisms with Breaks. Commun. Math. Phys. 320, 347-377, (2013).

[17] K. M. Khanin and A. Yu. Teplinsky. Herman's theory revisited. Invent. math., 178, 333-344, (2009).

[18] K. Khanin, E. Vul, Circle homeomorphisms with weak discontinuities. In proc. of Dynamical systems and statistical mechanics. 57-98, (Moscow, 1991). Amer. Math. Soc, Providence, RI, (1991). 
[19] K. Khanin, M. Yampolsky, Hyperbolicity of renormalization of circle maps with a break-type singularity. Moscow Mathematical Journal. 15,(1), 107-121,(2015).

[20] D. V. Khmelev, Rational rotation numbers for homeomorphisms with several breaktype singularities. Ergod. Th. \& Dynam. Sys. 25, 553-592, (2005).

[21] O. E. Lanford, Renormalization group methods for critical circle mapping. Nonlinear evolution and chaotic phenomena, NATO Adv. Sci. Inst. Ser. B: Phys., 176, 25$36,(1988)$.

[22] E. de Faria, W. de Melo, Rigidity of critical cirle maps I, J. Eur. Math. Soc. 1(4), 339-392, (1999).

[23] E. de Faria, W. de Melo, Rigidity of critical cirle maps II, Am. Math. Soc. 13(2), 343-370, (2000).

[24] W. de Melo and S. van Strien, One-dimensional Dynamics, Springer-Verlag, New York, (1995).

[25] J. Moser. A rapid convergent iteration method and non-linear differential equations. II. Ann. Scuola Norm. Sup. Pisa, 20(3), 499-535, (1966).

[26] J. Stark, Smooth coniugacy and renormalisation for diffeomorphisms of the circle. Nonlinearity 1, 541-575,(1988).

[27] D. Sullivan. Bounds, quadratical differentials and renormalization conjectures. Mathematics into Twenty-first Century, Vol.2. Providence, RI, Amer. Math. Soc., (1992).

[28] E. Vul and K. Khanin, Homeomorphisms of the circle with singularities of break type. Uspekhi Mat. Nauk, 45:3 (1990), 189190; English transl. Russian Math. Surveys, 45:3, 229-230, (1990).

[29] M. Weiss and A. Zygmund, A note on smooth functions. Indag. Math. 62, 52$58,(1959)$.

[30] M. Yampolsky, Hyperbolicity of renormalization of critical circle maps, Publ. Math. Inst. Hautes Sci. 96, 1-41, (2002).

[31] J.-C. Yoccoz, Conjugaison différentiable des difféomorphismes du cercle dont le nombre de rotation vérifie une condition diophantienne, Ann. Sci. École Norm. Sup. 417 (3), 333-359, (1984).

[32] A. Zygmund, Trigonometric series. Third Edition vol. I and II combined. Cambridge University Press. London, (2002). 